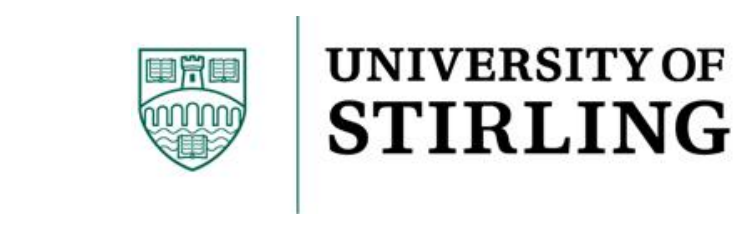

Stirling Management School

How wrong can you be? Implications of incorrect utility function specification for welfare measurement in choice experiments

Cati Torres

Nick Hanley

Antoni Riera

Stirling Economics Discussion Paper 2010-12

November 2010

Online at http://www.economics.stir.ac.uk 
How wrong can you be? Implications of incorrect utility function specification for welfare measurement in choice experiments.

\section{Cati Torres*, Nick Hanley $^{\perp}$, Antoni Riera*}

*Corresponding author. Centre de Recerca Econòmica (UIB. SA NOSTRA), 07122Palma de Mallorca, Spain. Email: cati.torres@uib.es. Telephone: +34 971171381.

${ }^{\perp}$ Division of Economics, University of Stirling, Stirling FK9 4LA, United Kingdom. Email: n.d.hanley@stir.ac.uk. Telephone: +44 1786466410.

*Centre de Recerca Econòmica (UIB- SA NOSTRA), 07122-Palma de Mallorca, Spain. Email: antoni.riera@uib.es. Telephone: +34 971171381. 


\title{
How wrong can you be? The implications of incorrect utility function specification for welfare measurement in choice experiments
}

\begin{abstract}
Despite the vital role of the utility function in welfare measurement, the implications of working with incorrect utility specifications have been largely neglected in the choice experiments literature. This paper addresses the importance of specification with a special emphasis on the effects of mistaken assumptions about the marginal utility of income. Monte Carlo experiments were conducted using different functional forms of utility to generate simulated choices. Multi-Nomial Logit and Mixed Logit models were then estimated on these choices under correct and incorrect assumptions about the true, underlying utility function. Estimated willingness to pay measures from these choice modelling results are then compared with the equivalent measures directly calculated from the true utility specifications. Results show that for the parameter values and functional forms considered, a continuous-quadratic or a discrete-linear attribute specification is a good option regardless of the true effects the attribute has on utility. We also find that mistaken assumptions about preferences over costs magnify attribute mis-specification effects.
\end{abstract}

Keywords: utility specification, attributes, welfare measurement, accuracy, efficiency, choice experiments, Monte Carlo analysis.

JEL classification: C51, D69, C99, C15. 


\section{Introduction}

Since the early 1990's, Choice Experiments (CE) have been increasingly used in the field of environmental valuation. One of their most important advantages is their ability to estimate willingness-to-pay (WTP) for changes in the attributes of an environmental good. In this context, decisions the researcher makes concerning the way in which these attributes are assumed to be related to utility are important. Decisions on how attributes enter the utility function involve which attributes to include, their nature (continuous or discrete) and the number of levels which attributes can take [1].

Given that attribute specification within the utility function assumed for a representative individual determines both the econometric approach taken to estimating choice models and the experimental design from which estimation proceeds, decisions over how to model the effects of changes in biodiversity, or air quality, or noise on utility may well have a significant impact on the accuracy and precision of marginal WTP estimates, and on the estimates of Compensating Surplus (CS) which can be derived from these. Decisions concerning the effects attributes have on utility are thus likely to be important, and an interesting question concerns the extent to which researchers produce inaccurate (biased) welfare measures by mistaken assumptions over the nature of the underlying utility function. Surprisingly, this question has not been addressed in the choice modelling literature.

Given that choice modellers must make decisions about the nature and the number of levels of attributes in a context of uncertain knowledge about the true preferences of individuals, this raises the question of the implications of working with incorrect utility specifications. As is well known, the parameter of the Cost attribute, usually interpreted 
as the negative of the marginal utility of income, plays a key role in welfare measurement. Specification issues related to the Cost variable include decisions over whether to assume a constant parameter or not. ${ }^{1}$ Problems related to the assumption of a non-constant Cost parameter $^{2}$ have led many researchers to assume it be a constant when specifying the utility function [2]. However, this is unlikely to be true in all cases [3]. Assuming as homogeneous a parameter that is likely to be heterogeneous could have important implications for welfare estimates. In this context, examining the sensitivity of attribute mis-specification effects should include mistaking assumptions about how the Cost parameter varies across individuals.

The objective of this paper is therefore to investigate the consequences of utility misspecification for $\mathrm{CE}$ estimates of welfare change, with a special emphasis on the effects of mistaken assumptions about the marginal utility of income. To do this, Monte Carlo (MC) experiments have been conducted where different attribute specifications and assumptions for both the Cost and an environmental quality parameter - that is, different functional forms of utility - have been used to generate simulated choices. Multi-Nomial Logit (MNL) and Mixed Logit (MXL) models have been estimated on these simulated choices under correct and incorrect assumptions about the true, underlying utility function (clearly, such tests are not available using real data, since the researcher cannot know the true form of utility). Inferred values for WTP from simulated choice data have then been compared with true WTP values obtained directly from the underlying utility specifications. This procedure has been repeated 1,000 times to examine the robustness of results.

\footnotetext{
${ }^{1}$ The Cost variable tends to enter the utility function as a continuous attribute in most CEs reported in the environmental economics literature.

${ }^{2}$ These problems include calculating implicit prices as a ratio of two distributions.
} 
The structure of the rest of this paper is as follows. The next section provides a review of the environmental valuation literature which has used Monte Carlo methods to assess specification, design and estimation issues. Section 3 discusses the methodology used and the data employed for the experiments. Results on attribute mis-specification, using one environmental attribute as an example, are reported in section 4 . In section 5 , the sensitivity of welfare measures to mistaken assumptions about the marginal utility of income is analysed. We also consider the implications of assuming constant error variances when this is not true. Next, a section on the errors in WTP involved from incorrectly assuming that the true, underlying utility function is additive is presented. Conclusions are drawn in section 7.

\section{Utility specification issues in the environmental valuation literature}

Despite the central role of utility function specification in welfare measurement, interest in choice modelling has thus far been restricted to the analysis of the impacts of alternative experimental designs under different utility specifications. Indeed, surprisingly little attention has been paid to utility specification issues in research on other valuation approaches based on random utility models, such as recreation demand models and dichotomous choice contingent valuation.

Recognition of the need for analyzing the accuracy of welfare estimates [4; 5] led researchers to investigate issues such as the specification of the recreation demand function in travel costs (TC) models, and WTP elicitation in the contingent valuation (CV) approach. These early studies were largely concerned with the factors affecting welfare measurement in revealed preference methods and dealt with the effects of different approaches to TC modelling. Thus, Kling [6] looks at the impacts on WTP 
estimates for quality changes in the Chesapeake Bay from the use of four different recreation demand models. Parameters from recreation surveys are combined with a utility function to simulate a TC data set to which the four alternative approaches are applied. Kling [6] finds that all approaches underestimate the true mean welfare change. A related paper is Kling [7], who again uses MC analysis to compare three different true utility specifications with alternative functional forms for the demand function in TC models. The paper shows that rather simple specifications for TC models can actually yield relatively small errors in welfare estimation.

Issues of functional form choice in TC models are also central to papers by Adamowicz et al [8] and Kling [9]. The former article looks at effects on the variance of welfare estimates, comparing linear, semilog, $\log -\log$ and restricted Box-Cox forms, and finds that impacts on both variance and mean can be substantial. In the latter paper, Kling [9] focuses on the magnitude of errors in WTP estimates from incorrect choice of functional form and finds that the choice of functional form is less important for small than big price changes. A related area of concern is decisions over appropriate nesting structures in multiple site recreation demand models. Kling \& Thomson [10] show that parameter estimates depend on both nesting structure and estimation method (sequential or Full Information Maximum Likelihood), whilst Herriges \& Kling [11] report the sign and size of bias from inappropriate nesting structures and analyze the ability of conventional goodness-of-fit tests to identify the best model.

Concerns about the accuracy and precision of welfare estimates can also be found in $\mathrm{CV}$. Thus, Kling [12] uses MC analysis to investigate the advantages of combining TC and CV data in terms of the bias and precision of welfare measures, and Alberini [13] 
analyzes, by undertaking MC experiments, the gains from using a double-bounded discrete choice model in the $\mathrm{CV}$ context, relative to a bivariate probit model and finds the double-bounded approach produces gains in terms of lower bias and greater precision. Scarpa \& Bateman [14] also use MC methods to analyse the design of followup questions in multiple-bounded question formats, and to investigate the efficiency gains from asking such follow-up questions, whereas Park et al [15] investigate the effects of functional form on WTP estimates within a discrete choice set-up.

Turning specifically to specification issues in CEs, many studies using simulation have been focused on the effects of different experimental design strategies. Thus, Ferrini \& Scarpa [16] use MC analysis to compare simple, shifted (orthogonal) designs with Defficient designs and cast light on both the use of prior information in undertaking experimental design, and the issue of whether the nature of the actual data generating process is consistent with that assumed by the analyst in choosing their econometric approach. Scarpa \& Rose [17] also analyze the performance of different design strategies, undertaken under the assumption that a prior belief on the range of values for the utility parameters can plausibly be defined, with a focus on efficiency of WTP estimates from a MNL model. Carlsson \& Martinsson [18] use MC analysis to compare three kinds of experimental design (orthogonal, cyclical and D-optimal) in terms of bias and mean squared error for three different true utility functions. In a similar vein is a paper by Lusk \& Norwood [19] who also use MC experiments to compare the effects of specifying utility as a continuous function of attributes, with a step-wise specification, in terms of the implications of alternative experimental designs. Their main finding is that true and estimated WTP are insignificantly different for all experimental designs 
considered, and that higher sample sizes always improve the fit of actual and estimated WTP.

A conclusion reached from this review is that the question of how important the specification of the utility function is for welfare measurement in CEs has received little attention. Given the key role of utility specification in welfare calculation, efforts need to be made to fulfil this gap. Indeed, some authors have argued that addressing the effects of misspecifying the underlying utility function - for example, using a linear form when true utility is non-linear - is an important area for future research [19].

\section{Designing MC experiments to examine the importance of attribute specification}

\subsection{The experimental design}

The attribute data employed to create the experimental design used in this paper come from a CE study on recreational beach use in Santa Ponça Bay, a small Mallorcan tourism area. ${ }^{3}$ We consider three non-monetary, environmental attributes $\left(X_{1}, X_{2}\right.$ and $\left.X_{3}\right)$ and a cost attribute $\left(X_{4}\right)$ varying at 3 levels each. ${ }^{4}$ The design has been generated under a D-efficiency criterion. As explained by Lusk \& Norwood [19], D-efficiency is a common measure of design efficiency representing a function of the geometric mean of the eigen values of $\left(X^{\prime} X\right)^{-1}$. It is formally given by $100 \cdot 1 / N\left|\left(X^{\prime} X\right)^{-1}\right|^{1 / A}$, where $X$ is the matrix of attributes used in the design, $N$ is the number of observations in a design

\footnotetext{
${ }^{3}$ For a detailed description of the attributes and their levels, see [20].

${ }^{4}$ Two variations on the levels of $X_{1}$ have also been considered to examine the effects of changing the number of levels: the first one with 2 levels and the second one with 5 levels. However, 2 levels only serve to identify a unique utility jump. Thus, the resulting attribute values have been the same under all the assumed attribute specifications regardless of the true effects $X_{1}$ has on utility. On the other hand, assigning 5 levels to $X_{1}$-a rather seldom practice in the literature- has led to similar results as those derived from assigning 3 levels. For all these reasons, these results on varying attribute levels have not been reported in the paper.
} 
and $A$ is the number of attribute $\mathrm{x}$ levels in the design. The design has also been generated allowing for main effects (ME) only. According to Louviere et al. [21], this kind of design typically explains about $70-90 \%$ of the variance in choice. The final design consisted of 36 pairs of attribute combinations. These have been then blocked into different versions each of 6 choice sets of 2 alternatives plus a business-as-usual (BAU) option. The main features of the design are shown in Table $1 .^{5}$

\subsection{Underlying utility functions and true welfare measures}

At the first stage of the MC analysis, three different generic utility functions with the same explanatory variables $\left(X_{1}, X_{2}, X_{3}\right.$ and $\left.X_{4}\right)$ and known parameters have been specified. The effects of utility function mis-specification are examined for the attribute $X_{1}$, which represented a measure of water quality in the CE. Thus, for a scenario in which $X_{1}$ has a true linear effect, a linear specification has been employed (Equation 1). To consider non-linear effects two different specifications have been used: a quadratic form (Equation 2); and a step-wise function (Equation 3) where the marginal utility of $X_{1}$ takes three constant values between 0 and $c_{2}{ }^{6}$

$U_{j i}=\alpha_{1} X_{j 1}+\beta X_{j 2}+\phi X_{j 3}+\omega X_{j 4}+\varepsilon_{j i}$

\footnotetext{
${ }^{5}$ A number of 36 pair combinations has been the result from application of a SAS. Given the BAU levels have been considered constant across the choice sets, only pair combinations have been optimized when creating the design. The BAU alternative has been added to the generated choice sets after the optimization process. However, the BAU levels (except $€ 0$ level for the Cost attribute) have not been for the exclusive use of the BAU option. Therefore, they have also been employed to generate the optimized pair combinations, this leading to a $3^{4}$ experimental design for each of the two alternatives.

${ }^{6}$ These utility function specifications are the most widely used in the literature when specifying an estimation model. In this context, if we consider researchers expect their utility specifications to fit well the data, it seems reasonable to use them to generate choices. Note that a utility specification matching Equation (3) for estimation purposes is given by a utility function where $X_{j 1}$ is codified as discrete by creating two dummy variables. Thus, if we want to identify the utility changes $\alpha_{1}$ and $\alpha_{2}$ with respect to when $X_{j 1} \geq c_{2}$, the utility specification would be as follows:
}

$U_{j i}=\alpha_{1} 1\left(X_{j 1}<c_{1}\right)+\alpha_{2} 1\left(c_{1} \leq X_{j 1}<c_{2}\right)+\beta X_{j 2}+\phi X_{j 3}+\omega X_{j 4}+\varepsilon_{j i}$ 
$U_{j i}=\alpha_{1} X_{j 1}+\alpha_{2} X_{j 1}^{2}+\beta X_{j 2}+\phi X_{j 3}+\omega X_{j 4}+\varepsilon_{j i}$

$U_{j i}=\alpha_{1}\left(X_{j 1}^{<}<c_{1}\right)+\alpha_{2}\left(c_{1} \leq X_{j 1}<c_{2}\right)+\alpha_{3}\left(X_{j 1} \geq c_{2}\right)+\beta X_{j 2}+\phi X_{j 3}+\omega X_{j 4}+\varepsilon_{j i}$

where $U_{j i}$ is the indirect utility of alternative $j$ for individual $i, \alpha_{1}, \alpha_{2}, \alpha_{3}, \beta, \phi, \omega$ are the known parameters of the attributes ( $\omega$ is the marginal utility of income), $c_{1}$ and $c_{2}$ are the critical attribute values delimiting the three steps of the step-wise marginal utility of $X_{1}$ and $\varepsilon_{j i}$ is the error term associated with alternative $j$ and individual $i$.

Following Hanemann [22], the true CS value of $X_{1}$, defined as the WTP for a change in the attribute from the BAU scenario, has been calculated for the linear, quadratic and step-wise utility specifications as shown in Equations (4), (5) and (6):

$$
\begin{aligned}
& C S=-\frac{1}{\omega}\left[\alpha_{1}\left(X_{1_{1}}-X_{1_{0}}\right)\right] \\
& C S=-\frac{1}{\omega}\left[\alpha_{1}\left(X_{1_{1}}-X_{1_{0}}\right)+\alpha_{2}\left(X_{1_{1}}^{2}-X_{1_{0}}^{2}\right)\right] \\
& C S=-\frac{1}{\omega}\left(\alpha_{y}-\alpha_{z}\right) ; \quad \mathrm{y}, \mathrm{z}=1,2,3
\end{aligned}
$$

where $X_{1_{1}}$ and $X_{1_{0}}$ are the policy-on and policy-off levels of $X_{1}$, respectively, and y and $\mathrm{z}$ represent one of the three ranges of the three-stepwise function and depend on the values of $c_{1}$ and $c_{2}$.

Table 2 shows the true utility specifications, the known parameters, the critical values $c_{1}$ and $c_{2}$ for the step-wise function and the true WTP values for a hypothetical change 
in $X_{1}$ from the BAU level (a value of 6 , as shown in Table 1) to a situation in which it takes the level 2, indicating a reduction in pollution. As seen in Table 2, two different quadratic specifications have been considered. These show two different degrees of non-linearity when $X_{1}$ has true quadratic effects on utility: a low degree (quadratic 1) and a high degree (quadratic 2). Using these two quadratic specifications will allow an examination of whether the intensity of true non-linearities has some influence on bias in the estimated attribute values.

\subsection{MC experiments and estimated welfare measures}

At the second stage of the analysis, MC experiments have been undertaken to estimate the CS value of the change in $X_{1}$ and compare it with the true value. Therefore, choices have been simulated for each type of true utility specification (linear, quadratic 1, quadratic 2 and step-wise). The utility of each alternative for each choice occasion has been calculated by combining the known parameters of the utility function with the attribute levels and an error term. These error terms have been generated from a type I extreme value distribution and a unique error has been randomly drawn not only for each alternative but also for each observation in the sample. ${ }^{7}$

This procedure generated 4 sets of simulated choices (one for each type of true utility function specification or data generating process). For each choice task, the simulated choice has been assigned to that alternative in the choice set providing the highest utility level. In the simulation, 600 individuals have been considered. Given each individual faces 6 choice tasks, 3,600 (600x6) observations have been created by this process for

\footnotetext{
${ }^{7}$ In an initial analysis, we tested the effects of changing the relative contribution of the error terms to total utility on the accuracy of welfare measures, but found that "small", "large" and "very large" error percentages gave rather similar accuracies for welfare measures. So, the main analysis in this new version of the paper uses just one error proportion- "small".
} 
each of the 4 data generating processes (DGP). Using these simulated samples, MNL models have then been estimated which encompass the range of true utility function specifications: that is, we estimate linear, quadratic and step-wise in $X_{1}$ MNL models.

Taking into account the types of true attribute specification (linear, quadratic 1, quadratic 2, stepwise) and attribute specification in the estimation model (continuouslinear, continuous-quadratic and discrete (step-wise)-linear), we have 12 different MC experiments (4x3). The CS value for the same change in $X_{1}$ has been estimated for each MC experiment following Equations (4), (5) and (6) according to the attribute specification assumed in the model. This process has been repeated 1,000 times, leading to a distribution of 1,000 estimated WTP values for the change in $X_{1}$ for each MC experiment. From each distribution, the mean WTP value of $X_{1}$ has been calculated as the average of the sum of the values obtained in each MC experiment over these 1,000 repetitions.

The importance of attribute specification has been examined by quantifying the errors in the estimated CS. To do this, bias and mean squared error (MSE) have been calculated. As shown in Equations (7) and (8), bias is defined as the average over 1,000 repetitions of the difference between the estimated and the true WTP for $X_{1}$. The MSE represents the average over 1,000 repetitions of the square of the bias and gives an idea of the variance of the estimates. ${ }^{8}$

\footnotetext{
${ }^{8}$ Note that $M S E=$ Bias $^{2}+\operatorname{var}\left(C S^{e}\right)$, where the variance is defined as the spread of the estimates about the mean of the estimates.
} 


$$
\begin{aligned}
& \text { BIAS }=\frac{1}{R}\left[\sum_{r=1}^{R}\left(C S_{r}^{e}-C S^{t}\right)\right] \\
& M S E=\frac{1}{R}\left[\sum_{r=1}^{R}\left(C S_{r}^{e}-C S^{t}\right)^{2}\right]
\end{aligned}
$$

where $R$ is the number of repetitions of each MC experiment, $C S_{r}^{e}$ is the estimated CS in repetition $r$ and $C S^{t}$ is the true $C S$.

Relative bias, defined as the ratio between our measure of bias and the true marginal value of $X_{1}$, has also been calculated to make comparable results from all the MC experiments. In what follows, we focus on relative bias as the measure of error in welfare estimation. ${ }^{9}$

\section{Results: mis-specifying how an environmental attribute affects utility.}

The results of relative bias in the estimated WTP value of $X_{1}$ for each MC experiment are presented in Table 3. As stated above, these values refer to a hypothetical change in $X_{1}$ from the BAU level of $X_{1}=6$ to a level of $X_{1}=2$. Relative bias measures are shown in terms of the true utility specification (linear, quadratic 1, quadratic 2, stepwise) and the specification assumed in the estimation model (continuous-linear, continuous-quadratic and discrete-linear) used in the simulations. We focus discussion on results where the assumed attribute specification does not match the true one.

As shown in Table 3, relative biases are highest when $X_{1}$ has true non-linear effects but a linear specification is assumed by the researcher. This is especially so when the

\footnotetext{
${ }^{9}$ In the interest of brevity, only results of relative bias are reported in the paper. Values for bias and MSE for all the MC experiments are provided in the Appendix.
} 
attribute has true quadratic effects with a marked degree of non-linearity (i.e. quadratic 2). In contrast, when $X_{1}$ has true linear effects and a quadratic or a discrete specification is erroneously assumed, relative biases are practically zero. That relative biases resulting from attribute misspecification increase when $X_{1}$ has true non-linear effects is not surprising as the linear specification is a particular case of the quadratic. Indeed, estimation results show that the quadratic model fits choice data from a true linear model well, yielding an insignificant squared parameter. In this context, and taking into account that preferences are in practice unknown, opting for a discrete or a quadratic attribute specification in estimation seems to be a good strategy when the researcher does not know the true underlying utility function. ${ }^{10}$

To analyze the sensitivity of results to the magnitude of welfare change, the MC experiments have been repeated considering a smaller attribute change than that used above. The analysis was repeated for a hypothetical change in $X_{1}$ from the BAU level (6, see Table 1) to a level of 4, rather than 2. Results show that for this smaller welfare change, attribute mis-specification when $X_{1}$ has true non-linear effects leads to less precise estimates than in the case for a bigger change in the attribute level. Again, when the attribute has true quadratic effects with a high degree of non-linearity the value of relative bias increases substantially when linear models are used. It seems then more risky to erroneously assume a linear specification when the welfare change considered is smaller. These results reinforce the idea that, in a context of uncertain preferences, the use of a quadratic or a discrete attribute specification is the best option.

\footnotetext{
${ }^{10}$ In particular, if the environmental attribute is quantitative, opting for a quadratic specification would probably lead to a better preference representation.
} 


\section{Mistaken assumptions about the Cost parameter}

The sensitivity of attribute mis-specification effects to mistaken assumptions about the marginal utility of income $(\omega)$ has been examined under the hypothesis that this parameter varies across individuals. In this context, for each true attribute specification (linear, quadratic 1, quadratic 2, step-wise, as used in the previous section), choices have been simulated. This has led to 4 sets of 600 simulated individuals. The utility of each alternative in each choice occasion has been calculated by combining the attribute levels with the known parameters of Table 2 except that of $\omega .{ }^{11}$ Heterogeneity of the marginal utility of income has been incorporated under the assumption that the Cost parameter is lognormally-distributed with a mean of 0.8 and a variance of $0.2 .^{12}$

To assess attribute mis-specification effects under mistaken assumptions about the marginal utility of income, two erroneous assumptions an analyst might make about the Cost parameter have been considered. First, we consider what happens when the analyst makes the assumption of homogeneity in $\omega$, when this is not true. Second, we consider the effects of assumptions concerning the distribution for $\omega$, where again the analyst "gets it wrong". As Balcombe et al. [3] note, there is considerable variation in the empirical literature about what distributional assumptions are made in MXL models. Empirically, this has been done by applying firstly a MNL model (reflecting a mistaken assumption that there is no variation in $\omega$ across respondents) and secondly a MXL model to the simulated choices. In the latter case, a triangular distribution has been

\footnotetext{
${ }^{11}$ To avoid problems related to the calculation of WTP values from the ratio of two distributions, the known parameters for the attributes other than Cost have been kept constant for all the simulated individuals. In other words, only heterogeneity in the true Cost parameter has been considered.

12 The authors are aware that this is one of the many ways to incorporate heterogeneity into the marginal utility of income when it randomly varies across individuals. Indeed, although it is not surprising that people value income differently at the margin, there is no certainty about the true distribution of the Cost parameter.
} 
assigned to $\omega$-this represents the analyst making the wrong distributional assumption. ${ }^{13}$ In each estimation model, $X_{1}$ has been codified as continuous-linear, continuousquadratic and discrete-linear to match equations (1), (2) and (3), respectively.

The results obtained by "incorrectly" applying the MNL and MXL models to the 4 sets of simulated choices have been compared with those derived from a correct application of the MXL model (i.e. where the analyst correctly assumes a lognormal distribution for $\omega$ to match the underlying DGP). Thus, three assumptions about $\omega$ have been considered: one correct and two erroneous. Across the possible types of true attribute specifications, assumed attribute specification and assumption about the Cost parameter there are 36 different $\mathrm{MC}$ experiments $(4 \times 3 \times 3)$. The $\mathrm{CS}$ of a change in $X_{1}$ from a level of 6 to a level of 2 has been estimated for each experiment following Equations (4), (5) and (6). Again, this process has been repeated 1,000 times leading to a distribution of 1,000 estimated WTP values for $X_{1}$ for each MC experiment. From each distribution, the WTP value of $X_{1}$ has been calculated as the average of the sum of the values obtained in each MC experiment over 1,000 repetitions. ${ }^{14}$

The results of relative bias in the estimated value of the change in $X_{1}$ for each of these MC experiments are presented in Table 4. Recall that these represent a hypothetical change in $X_{1}$ from the BAU level of 6 (Table 1) to a level of 2. As seen in Table 4,

\footnotetext{
${ }^{13}$ Like the lognormal distribution, the triangular distribution can be constrained to have the same sign for the parameter of interest. This is why it can also be assigned to a random Cost parameter when the lognormal distribution is not assumed. Given that the Matlab code by Kenneth Train to estimate the MXL model has been used in this paper, the triangular distribution for the Cost parameter has been defined as follows: $\omega \sim$ Triangular $(\mu+\sigma t)$ where $t$ is triangular between -1 and 1 , and $\mu$ and $\sigma$ are estimated.

${ }^{14}$ Like for the calculation of the simulated value of $X_{1}$, the estimated value for each MC experiment when the MXL model is applied has been obtained by averaging the sum of the individual marginal values over all the individuals of the sample.
} 
when the heterogeneity in the marginal utility of income is correctly captured by the researcher (third column), attribute misspecification always leads to higher relative biases. This is especially true when a linear specification is assumed in a context in which $X_{1}$ has true non-linear effects. In this case, the higher the non-linearity of the true quadratic effects, the less accurate the researcher's estimates.

Looking at the results from erroneously assuming that the Cost parameter is constant across respondents (fourth column), it is easy to see that decisions about attribute specification gain importance for welfare measurement. Indeed, all relative biases increase in magnitude in comparison with those of the third column. This indicates that mistakes over the presence of preference heterogeneity for costs magnify mistakes over functional form, in some cases by a factor of 3.5. Results show that, in general, WTP errors due to attribute misspecification are again higher when the attribute has true nonlinear effects. It is then generally worse to erroneously assume a linear specification than to erroneously assume a quadratic or a discrete one. Again, this is especially marked when $X_{1}$ has a true "quadratic 2" specification.

Finally, allowing for preference heterogeneity but with an incorrect distribution (fifth column) does not always lead to higher relative biases than those of the third column. In particular, this is so when $X_{1}$ has true quadratic effects and a linear specification is assumed. It would seem that mistaking the distribution of the Cost parameter partly compensates for the effects of attribute mis-specification in this case. Note that under an erroneous distributional assumption about $\omega$, codifying $X_{1}$ as continuous-linear is 
always advantageous when it has true non-linear effects. Likewise, attribute misspecification under true, linear effects seems to be a non-relevant issue.

By looking at results of Table 4, the importance of working under incorrect assumptions about the marginal utility of income is easily seen. In this sense, assuming this parameter to be constant when it actually varies across individuals is more critical than mistaking its distribution. Indeed, despite not always leading to higher relative biases, it makes attribute mis-specification a non-trivial issue in all cases.

The sensitivity of results on cost preference heterogeneity to the magnitude of the welfare change has also been examined. Again, the MC experiments have been repeated considering a hypothetical change in $X_{1}$ from the BAU level of 6 to a level of 4: that is, for a smaller change in environmental quality. In contrast to a high welfare change, values of relative bias show that mistaking the distribution of the Cost parameter makes relevant attribute mis-specification effects in all the cases. Indeed, it always leads to less precise estimates when $\omega$ is assigned a triangular distribution. On the other side, erroneously assuming constant the Cost parameter only makes attribute misspecification a non-trivial issue when $X_{1}$ has true, quadratic effects. Additionally, relative biases derived from a mis-specified $X_{1}$ increase with respect to a high welfare change in most of cases. This is especially so when it has true quadratic 2 effects, this reinforcing the appropriateness of opting for quadratic or discrete specifications. Therefore, results for a small welfare change show that working under incorrect assumptions about the marginal utility of income leads to higher attribute mis-specification effects in most of cases. In other words, the consequences of attribute mis-specification where preferences 
are heterogeneous seem to depend on the size of environmental quality change being valued.

\subsection{Overlooking scale heterogeneity in the Cost parameter}

In CEs, preference heterogeneity is commonly understood on the basis of MXL models. When the Cost parameter is that assumed to be heterogeneous, MXL assumptions imply the existence of a mean Cost attribute weight in the population. It is assumed each person-specific marginal utility of income represents the mean attribute weight plus the person-specific deviation from that mean. However, heterogeneity in $\omega$ could also be driven by scale heterogeneity. Indeed, some authors argue that true error variances are likely to be non-constant, thus explaining heterogeneity in attribute preferences [23].

The sensitivity of attribute mis-specification effects to mistaken assumptions about the marginal utility of income should then be analyzed under the hypothesis that the true heterogeneity in $\omega$ is also driven by a non-constant scale of the error. ${ }^{15}$ To do this, four sets of choices have been simulated following the DGP steps from section 5 but considering variation in the Cost parameter across individuals in the terms described in [23] for Generalized Multinomial Logit (G-MNL) models. That is, heterogeneity in $\omega$ has been considered as being caused by both scale heterogeneity (lognormallydistributed with 1 mean and 0.9 standard deviation) and lognormally-distributed mean heterogeneity (as described in section 5, with 0.8 mean and 0.2 standard deviation, independent of the variation induced by scale). ${ }^{16}$

\footnotetext{
15 The authors are grateful to an anonymous reviewer who suggested testing for this.

${ }^{16}$ G-MNL models are presented as nested structures combining both the MXL model and the scaleheterogeneity model [23]. According to this, the true Cost parameter can be written as $\omega_{i}=\sigma_{i}\left(\omega+\eta_{i}\right)=\sigma_{i} \omega+\eta_{i}^{*}$, where the random variable $\sigma_{i}$ captures scale heterogeneity and $\eta_{i}^{*}$ captures
} 
The assessment of attribute mis-specification effects under mistaken assumptions about the marginal utility of income has been focused on two types of erroneous assumptions. First, as in section 5, the assumption of homogeneity in $\omega$. Again, this has been empirically done by applying MNL models to the simulated choices. Second, an erroneous assumption about the source of heterogeneity in $\omega$, where the analyst overlooks scale heterogeneity. Here, MXL models have been applied by assigning both a lognormal and a triangular distribution to the Cost parameter to test for potential effects of distributional assumptions. Given MXL models assume the idiosyncratic error is independent and identically distributed extreme value, these models could be flawed in such cases leading to important implications for welfare measurement. In each estimation model, $X_{1}$ has again been codified as continuous-linear, continuousquadratic and discrete-linear to match Equations (1), (2) and (3), respectively. The true and assumed attribute specifications and the mistaken assumptions about $\omega$ have led to 36 different MC experiments (4x3x3), from which the CS of a high and a small change in $X_{1}$ has been calculated (as in previous sections).

Table 5 shows the relative biases in the estimated value of the high change in $X_{1}$ under each mistaken assumption about $\omega$.

When comparing these values with those from Table 4, one observes that decisions about attribute specification gain importance for welfare measurement. ${ }^{17}$ Indeed, all

residual taste heterogeneity. For the purpose of our paper, we have considered the standard deviation of $\eta_{i}^{*}$ is proportional to $\sigma_{i}$, that is, we have worked on the G-MNL-II model introduced in [23] (i.e. $\gamma=0$ ).

${ }^{17}$ By comparing Tables 4 and 5, we examine the performance of MNL and MXL model applications to choices simulated under DGPs only differing from the source of heterogeneity in the Cost parameter. Thus, we compare values from third and fourth columns in Table 5 with those from fourth and third columns in Table 4, respectively, and those from fifth columns in both tables. 
relative biases increase in magnitude in most of cases when the analyst mis-specifies the attribute. However, only under the erroneous assumptions of homogeneity in $\omega$ (third column) and a lognormal-distributed $\omega$ (fourth column) attribute mis-specification becomes a non-trivial issue. In these cases, errors in WTP from attribute misspecification are higher when $X_{1}$ has true, non-linear effects, this indicating again that quadratic and discrete specifications seem to be a good option when the researcher is uncertain. $^{18}$

Relative biases show that the loss of accuracy is especially marked when applying a MXL model under the assumption of a triangular distribution for the Cost parameter, followed by erroneously assuming it be a constant. ${ }^{19}$ Interestingly, comparisons of these values with those from Table 4 show that the lowest increases in relative biases occur under the application of a MXL model assuming a lognormally-distributed Cost parameter. This suggests that the relevance of mistaking the source of heterogeneity depends on the distributional assumptions for the Cost parameter. Indeed, under a scenario of scale heterogeneity in $\omega$, assuming constant error variances (i.e. erroneously applying MXL models) is less critical when the distribution assigned to $\omega$ matches that of the heterogeneity in $\omega$ independent of the variation induced by scale. Although a smaller welfare change leads in general to less accurate estimates, especially under the erroneous assumptions of homogeneity in $\omega$ and a lognormal-distributed $\omega$,

\footnotetext{
${ }^{18}$ Note that only assuming a quadratic specification under true, linear effects makes attribute misspecification a relevant issue when assigning a triangular distribution to the Cost parameter (fifth column).

${ }^{19}$ Results of MSE show a very high impact on the variance of the estimated welfare measures for these cases.
} 
only in few cases attribute mis-specification becomes a non-trivial issue. ${ }^{20}$ Thus, the accuracy losses related to a smaller welfare change seem to be compensated by the gains in terms of a less relevance of attribute specification.

\section{A note on mistaken additive assumptions about the true utility functional form}

Assuming a utility function which is additive in its attributes is common practice in CEs. $^{21}$ Our MC analysis has thus revolved around the implications for welfare measurement of erroneous assumptions about the true effects attributes have on utility on the basis of additive specifications. In this sense, we have opted for quadratic and step-wise functional forms to simulate choices from DGPs following non-linear attribute specifications. However, non-linear effects on utility can also be derived from non-additive attribute relationships. As true preferences are unknown, this raises the question of what the WTP errors from making the common additive assumptions would be if individuals used non-additive strategies to make choices. ${ }^{22}$ To shed light on this, we have repeated the analysis from previous sections using true, non-additive utility functions. $^{23}$

\footnotetext{
20 This happens under true, quadratic effects and estimation through a MXL model assuming a lognormally-distributed Cost parameter, and under true, linear effects and the assumption of a triangulardistributed Cost parameter when specifying $X_{1}$ in a quadratic way.

${ }^{21}$ This explains our focus on additive utility specifications both to simulate choices and to specify the estimation models in the MC experiments as well as our use of a ME only experimental design.

${ }^{22}$ Note that under DGPs following non-additive attribute specifications, linear, quadratic and discrete functional forms represent mistaken additive utility assumptions.

23 The authors are grateful to an anonymous reviewer who asked for testing for this. He argued that restricting the analysis within the belief system of additive utility specifications could be somehow limiting as individuals could also use non-additive strategies to make choices.
} 
In this sense, we have simulated choices under a utility specification where $X_{1}$ depicts a

Cobb-Douglas relationship with $X_{2}$, the remaining attributes $\left(X_{3}\right.$ and $\left.X_{4}\right)$ entering in an additive, linear way, as shown by Equation 9:

$U_{j i}=\alpha X_{j 1}^{\rho} X_{j 2}^{1-\rho}+\phi X_{j 3}+\omega X_{j 4}+\varepsilon_{j i}$

Table 6 shows the known parameters used and the true WTP values for the high change in $X_{1}$ from the BAU level (6) to a level of 2, at each level of $X_{2}$.

As seen, two types of Cobb-Douglas utility functions have been considered to test for the effects on WTP bias from mistaken additive attribute specifications of different values for $\rho$ (i.e. different contributions to utility of $X_{1}$ at each level of $X_{2}$, regardless of the value of $\alpha) .{ }^{24}$ Based on these known parameters and Equation (9), choices have been simulated according to the DGP steps described in previous sections and all the MC experiments repeated ${ }^{25}$.

\footnotetext{
${ }^{24}$ Technically, $\rho$ determines the slope of the indifference curves for $X_{1}$ and $X_{2}$. With $X_{1}$ in $\mathrm{Y}$ axis and $X_{2}$ in $\mathrm{X}$ axis, when $X_{1}$ and $X_{2}$ are goods lower values of $\rho$ imply the indifference curves are flatter and hence individuals are willing to give up less quantity of $X_{1}$ for one additional unit of $X_{2}$. This means the part-worth utility of $X_{1}$ is higher for lower values of $\rho$ at each level of $X_{1}$, increasing with the level of $X_{2}$. However, note that $\alpha$ is negative, indicating $X_{1}$ and $X_{2}$ act as bads, thus depicting a part-worth disutility from their Cobb-Douglas relationship. In this case, a lower value of $\rho$ implies a higher (lower) part-worth disutility (utility) of $X_{1}$ at each level of $X_{1}$, increasing (decreasing) with the level of $X_{2}$. Note that by using these values for $\rho$, we move away from common two-way attribute interactions considered sometimes in CEs. Additionally, note that the true value of the change in $X_{1}$ is higher under a Cobb-Douglas 1 utility function at each level of $X_{2}$. Indeed, given this function is steeper, it implies a higher variation of disutility between two given levels of $X_{1}$ and, hence, a higher WTP for a change in the attribute.

${ }^{25}$ The analyses leading to Tables 3,4 and 5 have been repeated under these true, non-additive utility functions. Note that the value 0.8 for the Cost parameter represents the mean of a lognormal distribution when it varies across individuals in the terms described in sections 5 and 5.1. Relative bias, bias and MSE for each MC experiment are reported in the Appendix at each level of $X_{2}$, both for a high and small welfare change in $X_{1}$.
} 
The analysis of the effects from mistaken additive utility assumptions when true preferences on cost are homogeneous shows that the level of $X_{2}$ is critical in determining the WTP errors. Despite this, the estimates are less accurate under a true Cobb-Douglas 2 utility specification at each level of $X_{2}$. Results also show a better performance of the linear specification in most cases, although the quadratic and discrete ones seems to be the best option for a smaller welfare change. Comparison of relative biases from mistaken additive assumptions under DGPs based on both additive and non-additive utility functional forms leads to an interesting conclusion. It shows that erroneously assuming linear, quadratic and discrete specifications does not necessarily imply less accurate estimates if the DGP follows a non-additive scheme. Indeed, cases can be found where erroneously assuming an additive attribute specification gives a lower WTP bias under a true, Cobb-Douglas utility functional form. The role of $\rho$ and $X_{2}$ is then critical in determining the relevance of mistaken additive assumptions. In other words, it is the type of non-additive relationship between attributes and not the simple fact that the true, utility function is non-additive which counts.

This conclusion above can also be drawn from the analysis of the sensitivity of the effects from mistaken additive specifications to mistaken assumptions about the marginal utility of income. In contrast, the quadratic and discrete specifications seem to be better for a higher welfare change. Similarly as in previous sections, when the true Cost parameter is lognormally-distributed, the mistaken assumptions of homogeneity and distribution magnify the WTP errors derived from mistakenly considering additive specifications. In this sense, the level of $X_{2}$ is critical in determining which mistaken assumption about the marginal utility of income leads to less accurate estimates. 
Likewise, erroneous assumptions about the Cost parameter when it is also driven by scale heterogeneity also make higher WTP bias. In addition, results from this analysis show again that the relevance of mistaking the source of heterogeneity in the Cost parameter (i.e. assuming constant error variances) depends on distributional assumptions. In other words, overlooking scale heterogeneity in the Cost parameter seems to be not so critical when the distributional assumption of deterministic effects matches that of the underlying true heterogeneity, independent of scale-induced variation.

\section{Conclusions}

When choice experimenters set out to value changes in environmental goods, they need to make assumptions about the utility function of people whose preferences they are trying to estimate. Researchers need to do this both with regard to constructing an experimental design and with regard to choice model estimation (these issues are of course closely linked with each other). However, it has been unclear to date how much bias can be introduced to welfare estimation by making the wrong assumptions: wrong assumptions on functional form, and wrong assumptions on preference heterogeneity. By applying MC analysis, this paper has investigated the importance of the specification of environmental attributes in the utility function for estimating their marginal value. Moreover, given that the Cost parameter plays a key role in welfare measurement, we have also investigated the sensitivity of attribute mis-specification effects to mistaking assumptions about the existence of heterogeneity in preferences towards cost, the distribution of preferences, and the source of heterogeneity. Results show that attribute mis-specification leads to mistakes in welfare estimates, especially in a context in which the attribute has true non-linear effects, both additive and non-additive. In this sense, the 
type of non-linearity is critical in determining the magnitude of bias. Indeed, the higher the true quadratic effects or the lower the value of $\rho$, the greater the potential bias in welfare measurement from mis-specification.

Mistaken assumptions about the Cost parameter can amplify environmental attribute mis-specification effects. This seems to be mainly so for assuming a constant parameter on cost, rather than getting the distribution wrong, although results are sensitive to size of the environmental changes being valued. When the Cost parameter is also driven by scale heterogeneity, distributional assumptions when applying a MXL model determine if erroneously assuming homogeneity is more critical than mistaking the source of heterogeneity (i.e. assuming constant error variances). Interestingly, results show that not accounting for scale heterogeneity is not so relevant when the distribution assigned to the Cost parameter matches that of its heterogeneity, independent of the scaleinduced variation.

Taking all results together, it would seem as though opting for quadratic or step-wise utility functions leads to the lowest overall degree of relative bias, compared to linear forms. Robustness to this is given by fact that additive attribute specifications do not necessarily lead to less accurate estimates if individuals use non-additive strategies to make choices. Yet linear utility functions are very common in choice models reported in the literature to date.

These results, however, are subject to the data employed in these MC experiments. That is, our conclusions are possibly specific to the experimental design, true utility attribute specifications, known parameters, error structures, and attribute specifications assumed 
in our models, and the assumptions about the marginal utility of income considered here. Although this suggests results may not be generalizable to all cases, the experimental design and methods of analysis employed here as "exemplars" are hardly un-common. Indeed, continuous and discrete non-monetary attributes with 3 levels and main effects only designs are features that can be found in many CE studies reported in the literature. Additionally, the analysis of the effects derived from erroneously assuming constant a Cost parameter that actually varies across individuals is an interesting result. It provides evidence of the magnitude of bias that can result when models are specified, as traditionally done, under the assumption of a constant marginal utility of income.

In a context in which utility specification issues have been largely overlooked in stated preference studies, this paper is only a first step on the long path to fill this gap. Although our results seem promising, it would be interesting to analyze the sensitivity of findings to different values of the known parameters. Likewise, the results for alternative attribute specifications, consideration of more than one non-monetary attributes varying across utility specifications, and experimental designs constructed on different efficiency criteria or allowing for interaction effects remain to be tested. Further research on these issues could help to examine the robustness of the conclusions drawn here. This will contribute to testing issues that, although being at the core of discrete choice studies, have been largely ignored in the literature to date. 


\section{References}

[1] D.A. Hensher, J.M. Rose, and W.H. Greene, Applied choice analysis: a primer, Cambridge University Press, Cambridge, UK, 2005.

[2] K. Train, and M. Weeks, Discrete choice models in preference space and willingness-to-pay space. in: R. Scarpa, and A. Alberini, (Eds.), Applications of simulation methods in environmental and resource economics, Springer, Dordrecht, The Netherlands, 2005, pp. 1-16.

[3] K. Balcombe, A. Chalak, and I. Fraser, Model selection for the mixed logit with Bayesian estimation. J. Environ. Econ. Manag. 57 (2009) 226-237.

[4] C.L. Kling, Estimating the precision of welfare measures. J. Environ. Econ. Manag. 21 (1991) 244-259.

[5] C.L. Kling, and R.J. Sexton, Bootstrapping in applied welfare analysis. Amer. J. Agr. Econ. 72 (1990) 406-418.

[6] C.L. Kling, A simulation approach to comparing multiple site recreation demand models using Chesapeake Bay survey data. Marine Resource Econ. 4 (1987) 95109.

[7] C.L. Kling, The reliability of estimates of environmental benefits from recreation demand models. Amer. J. Agr. Econ. 70 (1988) 892-901.

[8] W.L. Adamowicz, J.J. Fletcher, and T. Graham-Tomasi, Functional form and the statistical properties of welfare measures. Amer. J. Agr. Econ. 71 (1989) 414421.

[9] C.L. Kling, The importance of functional form in the estimation of welfare. Western J. Agr. Econ. 14 (1989) 168-174.

[10] C.L. Kling, and C.J. Thomson, The implications of model specification for welfare estimation in nested logit models. Amer. J. Agr. Econ. 78 (1996) 103114.

[11] J.A. Herriges, and C.L. Kling, The performance of nested logit models when welfare estimation is the goal. Amer. J. Agr. Econ. 79 (1997) 792-802.

[12] C.L. Kling, The gains from combining travel cost and contingent valuation data to value nonmarket goods. Land Econ. 73 (1997) 428-439.

[13] A. Alberini, Efficiency vs bias of willingness-to-pay estimates: Bivariate and interval-data models. J. Environ. Econ. Manag. 29 (1995) 169-180.

[14] R. Scarpa, and I. Bateman, Efficiency gains afforded by improved bid designs versus follow-up valuation questions in discrete-choice CV studies. Land Econ. 76 (2000) 299-311.

[15] T. Park, J.B. Loomis, and M. Creel, Confidence intervals for evaluating benefits estimates from dichotomous choice contingent valuation studies. Land Econ. 67 (1991) 64-73.

[16] S. Ferrini, and R. Scarpa, Designs with a priori information for nonmarket valuation with choice experiments: A Monte Carlo study. J. Environ. Econ. Manag. 53 (2007) 342-363.

[17] R. Scarpa, and J.M. Rose, Design efficiency for non-market valuation with choice modelling: how to measure it, what to report and why. Australian J. Agr. Resource Econ. 52 (2008) 253-282.

[18] F. Carlsson, and P. Martinsson, Design techniques for stated preference methods in health economics. Health Econ. 12 (2003) 281-294.

[19] J.L. Lusk, and F.B. Norwood, Effect of experimental design on choice-based conjoint valuation estimates. Amer. J. Agr. Econ. 87 (2005) 771-785. 
[20] C.M. Torres, A. Riera, and D. García, Are preferences for water quality different for second-home residents? Tourism Econ. 15 (2009) 629-651.

[21] J.J. Louviere, D.A. Hernsher, and J.D. Swait, Stated choice methods: analysis and application., Cambridge University Press, Cambridge, UK, 2000.

[22] W.M. Hanemann, Applied welfare analysis with quantitative response models, Working paper no. 241. University of California, Berkeley, 1984.

[23] D.G. Fiebig, M.P. Keane, J. Louviere, and N. Wasi, The generalized multinomial logit model: Accounting for scale and coefficient heterogeneity. Marketing Science. Articles in Advance (2009) 1-29. 
Table 1. Features of the experimental design

\begin{tabular}{|c|c|c|}
\hline \multicolumn{2}{|c|}{$\begin{array}{l}\text { Experimental design } \\
\text { factors }\end{array}$} & Design \\
\hline Attribute levels & $\begin{array}{l}\mathbf{X}_{1} \\
\mathbf{X}_{2} \\
\mathbf{X}_{3} \\
\mathbf{X}_{4}\end{array}$ & $\begin{array}{llll}2 & 4 & 6^{*} & \\
3 & 6 & 8^{*} & \\
0.3 & 1^{*} & 2 \\
3 & 10.5 & 24 & \left(0^{*}\right)\end{array}$ \\
\hline \multirow{3}{*}{\multicolumn{2}{|c|}{$\begin{array}{l}\text { Alternatives } \\
\text { Choice sets per individual } \\
\text { Blocks }\end{array}$}} & $2+\mathrm{BAU}$ \\
\hline & & 6 \\
\hline & & 6 \\
\hline \multicolumn{2}{|c|}{ Block replications } & 100 \\
\hline \multicolumn{2}{|c|}{ Total observations } & 3,600 \\
\hline
\end{tabular}

*Starred numbers correspond to the levels for the BAU option.

${ }^{a}$ Total observations are the number of choice sets $\mathrm{x}$ the number of blocks $\mathrm{x}$ the number of block replications. 
Table 2. True utility specifications and true CS

\begin{tabular}{ccccc}
\hline Parameters & Linear & Quadratic 1 & Quadratic 2 & 3-Stepwise \\
\hline$\alpha_{1}$ & -2.1 & -2 & -5.5 & -4.5 \\
$\alpha_{2}$ & & 0.1 & 0.5 & -9 \\
$\alpha_{3}$ & & & & -13 \\
$\beta$ & -0.7 & -0.7 & -0.7 & -0.7 \\
$\phi$ & 0.4 & 0.4 & 0.4 & 0.4 \\
$\omega$ & -0.8 & -0.8 & -0.8 & -0.8 \\
$c_{1}$ & & & & 3 \\
$c_{2}$ & & & & 5 \\
$\begin{array}{c}\text { True value of } \\
\text { the change in } \mathbf{X}_{\mathbf{1}}\end{array}$ & 10.5 & 6 & 7.5 & 10.6 \\
\hline
\end{tabular}


Table 3. Relative bias in the estimated value of a hypothetical change in $X_{1}$ (over 1,000 repetitions)

\begin{tabular}{llc}
\hline $\begin{array}{l}\text { True utility } \\
\text { Specification }\end{array}$ & $\begin{array}{l}\text { Assumed utility } \\
\text { specification }\end{array}$ & Relative bias \\
\hline \multirow{2}{*}{ Linear } & Linear & -0.0002 \\
& Quadratic & -0.0003 \\
& Discrete & -0.0003 \\
\hline \multirow{3}{*}{ Quadratic 1 } & Linear & -0.0245 \\
& Quadratic & -0.0001 \\
& Discrete & -0.0001 \\
\hline \multirow{2}{*}{ Quadratic 2 } & Linear & -0.0674 \\
& Quadratic & 0.0002 \\
& Discrete & 0.0002 \\
\hline \multirow{3}{*}{ 3-Stepwise } & Linear & -0.0119 \\
& Quadratic & -0.0008 \\
& Discrete & -0.0008 \\
\hline
\end{tabular}


Table 4. Relative bias in the estimated value of a change in $X_{1}$ under each assumption about $\omega$ when the true Cost parameter is lognormally-distributed (over 1,000 repetitions)

\begin{tabular}{|c|c|c|c|c|}
\hline \multirow{2}{*}{$\begin{array}{l}\text { True utility } \\
\text { specification }\end{array}$} & \multirow{2}{*}{$\begin{array}{l}\text { Assumed utility } \\
\text { specification }\end{array}$} & \multirow{2}{*}{$\begin{array}{c}\text { Correct } \\
\text { assumption } \\
\text { Lognormal }\end{array}$} & \multicolumn{2}{|c|}{ 'Mistaken assumptions' } \\
\hline & & & Constant & Triangular \\
\hline \multirow{3}{*}{ Linear } & Linear & -0.0148 & -0.0570 & 0.1133 \\
\hline & Quadratic & -0.0170 & -0.0604 & 0.1134 \\
\hline & Discrete & -0.0170 & -0.0604 & 0.1135 \\
\hline \multirow{3}{*}{ Quadratic 1} & Linear & -0.0394 & -0.0655 & 0.0259 \\
\hline & Quadratic & -0.0148 & -0.0474 & 0.0580 \\
\hline & Discrete & -0.0148 & -0.0474 & 0.0580 \\
\hline \multirow{3}{*}{ Quadratic 2} & Linear & -0.0868 & -0.1104 & -0.0297 \\
\hline & Quadratic & -0.0134 & -0.0590 & 0.0487 \\
\hline & Discrete & -0.0134 & -0.0590 & 0.0487 \\
\hline \multirow{3}{*}{ 3-Stepwise } & Linear & -0.0297 & -0.0677 & 0.0846 \\
\hline & Quadratic & -0.0178 & -0.0610 & 0.1142 \\
\hline & Discrete & -0.0178 & -0.0610 & 0.1153 \\
\hline
\end{tabular}


Table 5. Relative bias in the estimated value of a change in $X_{1}$ under each assumption about $\omega$ when the true Cost parameter presents scale heterogeneity (over 1,000 repetitions)

\begin{tabular}{lllcc}
\hline True utility & Assumed utility & & \multicolumn{2}{c}{ Mistaken heterogeneity source } \\
\cline { 5 - 5 } specification & specification & Constant & Lognormal & Triangular \\
\hline \multirow{2}{*}{ Linear } & Linear & -0.4943 & -0.0380 & -0.4470 \\
& Quadratic & -0.4941 & -0.0390 & -2.6501 \\
& Discrete & -0.4941 & -0.0390 & 0.0628 \\
\hline \multirow{3}{*}{ Quadratic 1 } & Linear & -0.5028 & -0.0759 & -0.7775 \\
& Quadratic & -0.4876 & -0.0536 & -1.1724 \\
& Discrete & -0.4876 & -0.0536 & -0.8075 \\
\hline \multirow{3}{*}{ Quadratic 2 } & Linear & -0.4978 & -0.0700 & -0.6105 \\
& Quadratic & -0.4753 & -0.0512 & -0.7724 \\
& Discrete & -0.4753 & -0.0512 & 1.0816 \\
\hline \multirow{3}{*}{ 3-Stepwise } & Linear & -0.4961 & -0.0431 & -0.6343 \\
& Quadratic & -0.4934 & -0.0377 & -1.0623 \\
& Discrete & -0.4934 & -0.0377 & -0.9978 \\
\hline
\end{tabular}


Table 6. True, non-additive utility specifications and true CS

\begin{tabular}{rrcc}
\hline \multicolumn{2}{c}{ Parameter values } & Cobb-Douglas 1 & Cobb-Douglas 2 \\
\hline \multicolumn{1}{c}{$\alpha$} & -2.1 & -2.1 \\
$\phi$ & & 0.4 & 0.4 \\
$\omega$ & & -0.8 & -0.8 \\
$\quad \rho$ & & 0.8 & 0.2 \\
True value & $\mathbf{X}_{\mathbf{2}}=\mathbf{8}$ & 9.76 & 3.91 \\
of the & $\mathbf{X}_{\mathbf{2}}=\mathbf{6}$ & 9.21 & 3.11 \\
change in & $\mathbf{X}_{\mathbf{2}}=\mathbf{3}$ & 8.02 & 1.78 \\
$\mathbf{X}_{\mathbf{1}}$ & & & \\
\hline
\end{tabular}




\section{APPENDIX TO PAPER}

Bias in the estimated value of a hypothetical change in $X_{1}$ from the BAU level (6) to a level of 2 (over 1,000 repetitions)

\begin{tabular}{llc}
\hline $\begin{array}{l}\text { True utility } \\
\text { specification }\end{array}$ & $\begin{array}{l}\text { Assumed utility } \\
\text { specification }\end{array}$ & Bias \\
\hline \multirow{3}{*}{ Linear } & Linear & -0.0025 \\
& Quadratic & -0.0034 \\
& Discrete & -0.0034 \\
\hline \multirow{3}{*}{ Quadratic 1 } & Linear & -0.1467 \\
& Quadratic & -0.0005 \\
& Discrete & -0.0005 \\
\hline \multirow{3}{*}{ Quadratic 2 } & Linear & -0.5059 \\
& Quadratic & 0.0016 \\
& Discrete & 0.0016 \\
\hline \multirow{3}{*}{ 3-Stepwise } & Linear & -0.1269 \\
& Quadratic & -0.0081 \\
& Discrete & -0.0081 \\
\hline
\end{tabular}

MSE in the estimated value of a hypothetical change in $\mathrm{X}_{1}$ from the BAU level (6) to a level of 2 (over 1,000 repetitions)

\begin{tabular}{llc}
\hline $\begin{array}{l}\text { True utility } \\
\text { specification }\end{array}$ & $\begin{array}{l}\text { Assumed utility } \\
\text { specification }\end{array}$ & MSE \\
\hline \multirow{3}{*}{ Linear } & Linear & 0.0291 \\
& Quadratic & 0.0316 \\
& Discrete & 0.0316 \\
\hline \multirow{3}{*}{ Quadratic 1 } & Linear & 0.0363 \\
& Quadratic & 0.0167 \\
& Discrete & 0.0167 \\
\hline \multirow{3}{*}{ Quadratic 2 } & Linear & 0.2727 \\
& Quadratic & 0.0175 \\
& Discrete & 0.0175 \\
\hline \multirow{3}{*}{ 3-Stepwise } & Linear & 0.0448 \\
& Quadratic & 0.0318 \\
& Discrete & 0.0318 \\
\hline
\end{tabular}


Relative bias in the estimated value of a hypothetical change in $X_{1}$ from the BAU level (6) to a level of 4 (over 1,000 repetitions)

\begin{tabular}{llc}
\hline $\begin{array}{l}\text { True utility } \\
\text { specification }\end{array}$ & $\begin{array}{l}\text { Assumed utility } \\
\text { specification }\end{array}$ & $\begin{array}{c}\text { Relative } \\
\text { bias }\end{array}$ \\
\hline \multirow{3}{*}{ Linear } & Linear & -0.0002 \\
& Quadratic & -0.0004 \\
& Discrete & -0.0004 \\
\hline \multirow{3}{*}{ Quadratic 1 } & Linear & 0.1707 \\
& Quadratic & -0.0009 \\
& Discrete & -0.0009 \\
\hline \multirow{3}{*}{ Quadratic 2 } & Linear & 1.7977 \\
& Quadratic & -0.0017 \\
& Discrete & -0.0017 \\
\hline \multirow{3}{*}{ 3-Stepwise } & Linear & 0.0498 \\
& Quadratic & 0.0003 \\
& Discrete & 0.0003 \\
\hline
\end{tabular}

Bias in the estimated value of a hypothetical change in $\mathrm{X}_{1}$ from the BAU level (6) to a level of 4 (over 1,000 repetitions)

\begin{tabular}{llc}
\hline $\begin{array}{l}\text { True utility } \\
\text { specification }\end{array}$ & $\begin{array}{l}\text { Assumed utility } \\
\text { specification }\end{array}$ & Bias \\
\hline \multirow{3}{*}{ Linear } & Linear & -0.0012 \\
& Quadratic & -0.0021 \\
& Discrete & -0.0021 \\
\hline \multirow{3}{*}{ Quadratic 1 } & Linear & 0.4266 \\
& Quadratic & -0.0022 \\
& Discrete & -0.0022 \\
\hline \multirow{3}{*}{ Quadratic 2 } & Linear & 2.2471 \\
& Quadratic & -0.0021 \\
& Discrete & -0.0021 \\
\hline \multirow{3}{*}{ 3-Stepwise } & Linear & 0.2490 \\
& Quadratic & 0.0016 \\
& Discrete & 0.0016 \\
\hline
\end{tabular}


MSE in the estimated value of a hypothetical change in $\mathrm{X}_{1}$ from the BAU level (6) to a level of 4 (over 1,000 repetitions)

\begin{tabular}{lll}
\hline $\begin{array}{l}\text { True utility } \\
\text { specification }\end{array}$ & $\begin{array}{l}\text { Assumed utility } \\
\text { specification }\end{array}$ & MSE \\
\hline \multirow{2}{*}{ Linear } & Linear & 0.0073 \\
& Quadratic & 0.0178 \\
& Discrete & 0.0178 \\
\hline \multirow{3}{*}{ Quadratic 1 } & Linear & 0.1857 \\
& Quadratic & 0.0147 \\
& Discrete & 0.0147 \\
\hline \multirow{3}{*}{ Quadratic 2 } & Linear & 5.0535 \\
& Quadratic & 0.0197 \\
& Discrete & 00197 \\
\hline \multirow{3}{*}{ 3-Stepwise } & Linear & 0.0692 \\
& Quadratic & 0.0181 \\
& Discrete & 0.0181 \\
\hline
\end{tabular}


Bias in the estimated value of a hypothetical change in $X_{1}$ from the BAU level (6) to a level of 2 under each assumption about $\omega$ when the true Cost parameter is lognormally-distributed (over 1,000 repetitions)

\begin{tabular}{llccc}
\hline $\begin{array}{l}\text { True utility } \\
\text { specification }\end{array}$ & $\begin{array}{l}\text { Assumed utility } \\
\text { specification }\end{array}$ & $\begin{array}{c}\text { Correct } \\
\text { assumption } \\
\text { Lognormal }^{\mathbf{a}}\end{array}$ & $\begin{array}{c}\text { 'Mistaken } \\
\text { Constant }^{\mathbf{b}}\end{array}$ & $\begin{array}{c}\text { assumptions' } \\
\text { Triangular }^{\mathbf{a}}\end{array}$ \\
\hline \multirow{2}{*}{ Linear } & Linear & -0.2079 & -0.8003 & 1.5917 \\
& Quadratic & -0.2394 & -0.8482 & 1.5929 \\
& Discrete & -0.2395 & -0.8481 & 1.5943 \\
\hline \multirow{2}{*}{ Quadratic 1 } & Linear & -0.3162 & -0.5260 & 0.2077 \\
& Quadratic & -0.1191 & -0.3808 & 0.4658 \\
& Discrete & -0.1191 & -0.3808 & 0.4658 \\
\hline \multirow{2}{*}{ Quadratic 2 } & Linear & -0.8709 & -1.1083 & -0.2981 \\
& Quadratic & -0.1347 & -0.5917 & 0.4886 \\
& Discrete & -0.1347 & -0.5916 & 0.4886 \\
\hline \multirow{3}{*}{ 3-Stepwise } & Linear & -0.4228 & -0.9628 & 1.2025 \\
& Quadratic & -0.2533 & -0.8667 & 1.6228 \\
& Discrete & -0.2533 & -0.8666 & 1.6392 \\
\hline
\end{tabular}

${ }^{\text {a }}$ Heterogeneity in $\omega$ is modelled through a MXL model

${ }^{\mathrm{b}} \omega$ is erroneously considered constant, so a MNL model is estimated

MSE in the estimated value of a hypothetical change in $X_{1}$ from the BAU level (6) to a level of 2 under each assumption about $\omega$ when the true Cost parameter is lognormally-distributed (over 1,000 repetitions)

\begin{tabular}{|c|c|c|c|c|}
\hline $\begin{array}{l}\text { True utility } \\
\text { specification }\end{array}$ & $\begin{array}{l}\text { Assumed utility } \\
\text { specification }\end{array}$ & $\begin{array}{c}\text { Correct } \\
\text { assumption } \\
\text { Lognormal }^{\text {a }} \\
\end{array}$ & $\begin{array}{l}\text { 'Mistaken } \\
\text { Constant }^{b} \\
\end{array}$ & $\begin{array}{l}\text { Issumptions' } \\
\text { Triangular }^{\mathrm{a}} \\
\end{array}$ \\
\hline \multirow{3}{*}{ Linear } & Linear & 0.0959 & 0.6812 & 6.2945 \\
\hline & Quadratic & 0.1143 & 0.7613 & 31.7297 \\
\hline & Discrete & 0.1144 & 0.7613 & 31.5773 \\
\hline \multirow{3}{*}{ Quadratic 1} & Linear & 0.1280 & 0.3015 & 0.1174 \\
\hline & Quadratic & 0.0458 & 0.1716 & 0.2933 \\
\hline & Discrete & 0.0458 & 0.1716 & 0.2933 \\
\hline \multirow{3}{*}{ Quadratic 2} & Linear & 0.7892 & 1.2547 & 0.1756 \\
\hline & Quadratic & 0.0551 & 0.3817 & 0.3309 \\
\hline & Discrete & 0.0551 & 0.3816 & 0.3309 \\
\hline \multirow{3}{*}{ 3-Stepwise } & Linear & 0.2338 & 0.9697 & 19.9077 \\
\hline & Quadratic & 0.1245 & 0.7964 & 30.0161 \\
\hline & Discrete & 0.1245 & 0.7964 & 35.2770 \\
\hline
\end{tabular}

\footnotetext{
${ }^{\text {a }}$ Heterogeneity in $\omega$ is modelled through a MXL model

${ }^{\mathrm{b}} \omega$ is erroneously considered constant, so a MNL model is estimated
} 
Relative bias in the estimated value of a hypothetical change in $X_{1}$ from the BAU level (6) to a level of 4 under each assumption about $\omega$ when the true Cost parameter is lognormally-distributed (over 1,000 repetitions)

\begin{tabular}{llccc}
\hline $\begin{array}{l}\text { True utility } \\
\text { specification }\end{array}$ & $\begin{array}{l}\text { Assumed utility } \\
\text { specification }\end{array}$ & $\begin{array}{c}\text { Correct } \\
\text { assumption } \\
\text { Lognormal }^{\mathbf{a}}\end{array}$ & $\begin{array}{c}\text { 'Mistaken } \\
\text { Constant }^{\mathbf{b}}\end{array}$ & $\begin{array}{c}\text { assumptions' } \\
\text { Triangular }^{\mathbf{a}}\end{array}$ \\
\hline \multirow{2}{*}{ Linear } & Linear & -0.0148 & -0.0570 & 0.1133 \\
& Quadratic & -0.0085 & -0.0423 & 0.1245 \\
& Discrete & -0.0085 & -0.0423 & 0.1246 \\
\hline \multirow{2}{*}{ Quadratic 1 } & Linear & 0.1527 & 0.1214 & 0.2310 \\
& Quadratic & -0.0121 & -0.0953 & 0.0157 \\
& Discrete & -0.0121 & -0.0953 & 0.0157 \\
\hline \multirow{2}{*}{ Quadratic 2 } & Linear & 1.7396 & 1.6687 & 1.9109 \\
& Quadratic & -0.0120 & -0.3936 & -0.0390 \\
& Discrete & -0.0119 & -0.3935 & -0.0390 \\
\hline \multirow{3}{*}{ 3-Stepwise } & Linear & 0.0309 & -0.0095 & 0.1524 \\
& Quadratic & -0.0081 & -0.0431 & 0.1254 \\
& Discrete & -0.0081 & -0.0431 & 0.1265 \\
\hline
\end{tabular}

${ }^{\text {a }}$ Heterogeneity in $\omega$ is modelled through a MXL model

${ }^{\mathrm{b}} \omega$ is erroneously considered constant, so a MNL model is estimated

Bias in the estimated value of a hypothetical change in $X_{1}$ from the BAU level (6) to a level of 4 under each assumption about $\omega$ when the true Cost parameter is lognormally-distributed (over 1,000 repetitions)

\begin{tabular}{llccc}
\hline $\begin{array}{l}\text { True utility } \\
\text { specification }\end{array}$ & $\begin{array}{l}\text { Assumed utility } \\
\text { specification }\end{array}$ & $\begin{array}{c}\text { Correct } \\
\text { assumption } \\
\text { Lognormal }^{\text {a }}\end{array}$ & $\begin{array}{c}\text { 'Mistaken } \\
\text { Constant }^{\mathbf{b}}\end{array}$ & $\begin{array}{c}\text { assumptions' } \\
\text { Triangular }^{\mathbf{a}}\end{array}$ \\
\hline \multirow{2}{*}{ Linear } & Linear & -0.1039 & -0.4002 & 0.7959 \\
& Quadratic & -0.0598 & -0.2971 & 0.8746 \\
& Discrete & -0.0598 & -0.2971 & 0.8753 \\
\hline \multirow{2}{*}{ Quadratic 1 } & Linear & 0.5109 & 0.4060 & 0.7729 \\
& Quadratic & -0.0404 & -0.3189 & 0.0524 \\
& Discrete & -0.0404 & -0.3189 & 0.0524 \\
\hline \multirow{2}{*}{ Quadratic 2 } & Linear & 2.9096 & 2.7909 & 3.1960 \\
& Quadratic & -0.0200 & -0.6582 & -0.0652 \\
& Discrete & -0.0119 & -0.6582 & -0.0652 \\
\hline \multirow{3}{*}{ 3-Stepwise } & Linear & 0.2067 & -0.0633 & 1.0194 \\
& Quadratic & -0.0542 & -0.2880 & 0.8386 \\
& Discrete & -0.0541 & -0.2881 & 0.8464 \\
\hline
\end{tabular}

${ }^{\text {a }}$ Heterogeneity in $\omega$ is modelled through a MXL model

${ }^{\mathrm{b}} \omega$ is erroneously considered constant, so a MNL model is estimated 
MSE in the estimated value of a hypothetical change in $X_{1}$ from the BAU level (6) to a level of 4 under each assumption about $\omega$ when the true Cost parameter is lognormally-distributed (over 1,000 repetitions)

\begin{tabular}{llccc}
\hline $\begin{array}{l}\text { True utility } \\
\text { specification }\end{array}$ & $\begin{array}{l}\text { Assumed utility } \\
\text { specification }\end{array}$ & $\begin{array}{c}\text { Correct } \\
\text { assumption } \\
\text { Lognormal }^{\text {a }}\end{array}$ & $\begin{array}{c}\text { 'Mistaken } \\
\text { Constant }^{\mathbf{b}}\end{array}$ & $\begin{array}{c}\text { assumptions' } \\
\text { Triangular }^{\mathbf{a}}\end{array}$ \\
\hline \multirow{2}{*}{ Linear } & Linear & 0.0240 & 0.1703 & 1.5736 \\
& Quadratic & 0.0345 & 0.1159 & 8.8220 \\
& Discrete & 0.0345 & 0.1159 & 8.7641 \\
\hline \multirow{2}{*}{ Quadratic 1 } & Linear & 0.2680 & 0.1711 & 0.6159 \\
& Quadratic & 0.0266 & 0.1333 & 0.0398 \\
& Discrete & 0.0266 & 0.1333 & 0.0398 \\
\hline \multirow{2}{*}{ Quadratic 2 } & Linear & 8.4733 & 7.7957 & 10.2360 \\
& Quadratic & 0.0353 & 0.4765 & 0.0441 \\
& Discrete & 0.0353 & 0.4764 & 0.0441 \\
\hline \multirow{3}{*}{ 3-Stepwise } & Linear & 0.0565 & 0.0147 & 5.6546 \\
& Quadratic & 0.0339 & 0.1118 & 6.8939 \\
& Discrete & 0.0339 & 0.1118 & 8.0835 \\
\hline
\end{tabular}

\footnotetext{
${ }^{a}$ Heterogeneity in $\omega$ is modelled through a MXL model

b $\omega$ is erroneously considered constant, so a MNL model is estimated
} 
Bias in the estimated value of a hypothetical change in $X_{1}$ from the BAU level (6) to a level of 2 under each assumption about $\omega$ when the true Cost parameter presents scale heterogeneity (over 1,000 repetitions)

\begin{tabular}{|c|c|c|c|c|}
\hline \multirow{2}{*}{$\begin{array}{l}\text { True utility } \\
\text { specification }\end{array}$} & \multirow{2}{*}{$\begin{array}{l}\text { Assumed utility } \\
\text { specification }\end{array}$} & \multirow[b]{2}{*}{ Constant ${ }^{b}$} & \multicolumn{2}{|c|}{ Mistaken heterogeneity source } \\
\hline & & & Lognormal $^{\mathrm{a}}$ & Triangular $^{\mathrm{a}}$ \\
\hline \multirow{3}{*}{ Linear } & Linear & -19.2057 & -1.4768 & -17.3696 \\
\hline & Quadratic & -19.1984 & -1.5167 & -102.9669 \\
\hline & Discrete & -19.1984 & -1.5164 & 2.4399 \\
\hline \multirow{3}{*}{ Quadratic 1 } & Linear & -11.1626 & -1.6853 & -17.2622 \\
\hline & Quadratic & -10.8263 & -1.1885 & -26.0303 \\
\hline & Discrete & -10.8263 & -1.1891 & -17.9273 \\
\hline \multirow{3}{*}{ Quadratic 2} & Linear & -13.8144 & -1.9416 & -16.9421 \\
\hline & Quadratic & -13.1914 & -1.4222 & -21.4376 \\
\hline & Discrete & -13.1913 & -1.4223 & 30.0175 \\
\hline \multirow{3}{*}{ 3-Stepwise } & Linear & -19.5031 & -1.6941 & -24.9399 \\
\hline & Quadratic & -19.3969 & -1.4838 & -41.7674 \\
\hline & Discrete & -19.3969 & -1.4832 & -39.2300 \\
\hline
\end{tabular}

${ }^{\text {a }}$ Heterogeneity in $\omega$ is modelled through a MXL model

${ }^{\mathrm{b}} \omega$ is erroneously considered constant, so a MNL model is estimated

MSE in the estimated value of a hypothetical change in $\mathrm{X}_{1}$ from the BAU level (6) to a level of 2 under each assumption about $\omega$ when the true Cost parameter presents scale heterogeneity (over 1,000 repetitions)

\begin{tabular}{|c|c|c|c|c|}
\hline \multirow{2}{*}{$\begin{array}{l}\text { True utility } \\
\text { specification }\end{array}$} & \multirow{2}{*}{$\begin{array}{l}\text { Assumed utility } \\
\text { specification }\end{array}$} & \multirow[b]{2}{*}{ Constant $^{b}$} & \multicolumn{2}{|c|}{ Mistaken heterogeneity source } \\
\hline & & & Lognormal $^{\mathbf{a}}$ & Triangular $^{\mathrm{a}}$ \\
\hline \multirow{3}{*}{ Linear } & Linear & 368.965 & 5.141 & 165705.648 \\
\hline & Quadratic & 368.688 & 5.288 & 4635943.747. \\
\hline & Discrete & 368.687 & 5.289 & 593152.422 \\
\hline \multirow{3}{*}{ Quadratic 1} & Linear & 124.675 & 3.739 & 7246.187 \\
\hline & Quadratic & 117.283 & 2.329 & 67190.985 \\
\hline & Discrete & 117.283 & 2.330 & 15305.266 \\
\hline \multirow{3}{*}{ Quadratic 2} & Linear & 190.920 & 5.077 & 353936.979 \\
\hline & Quadratic & 174.090 & 3.218 & 10402.740 \\
\hline & Discrete & 174.090 & 3.218 & 3180365.102 \\
\hline \multirow{3}{*}{ 3-Stepwise } & Linear & 380.475 & 5.809 & 98446.925 \\
\hline & Quadratic & 376.342 & 5.180 & 21156.974 \\
\hline & Discrete & 376.342 & 5.178 & 185120.038 \\
\hline
\end{tabular}

${ }^{\text {a }}$ Heterogeneity in $\omega$ is modelled through a MXL model

${ }^{\mathrm{b}} \omega$ is erroneously considered constant, so a MNL model is estimated 
Relative bias in the estimated value of a hypothetical change in $X_{1}$ from the BAU level (6) to a level of 4 under each assumption about $\omega$ when the true Cost parameter presents scale heterogeneity (over 1,000 repetitions)

\begin{tabular}{|c|c|c|c|c|}
\hline \multirow{2}{*}{$\begin{array}{l}\text { True utility } \\
\text { specification }\end{array}$} & \multirow{2}{*}{$\begin{array}{l}\text { Assumed utility } \\
\text { specification }\end{array}$} & \multirow[b]{2}{*}{ Constant $^{b}$} & \multicolumn{2}{|c|}{ Mistaken heterogeneity source } \\
\hline & & & Lognormal $^{\mathbf{a}}$ & Triangular $^{\mathrm{a}}$ \\
\hline \multirow{3}{*}{ Linear } & Linear & -0.4943 & -0.0380 & -0.4470 \\
\hline & Quadratic & -0.4968 & -0.0312 & -2.7142 \\
\hline & Discrete & -0.4968 & -0.0312 & 0.0977 \\
\hline \multirow{3}{*}{ Quadratic 1} & Linear & -0.4033 & 0.1089 & -0.7330 \\
\hline & Quadratic & -0.6297 & -0.0524 & -1.1745 \\
\hline & Discrete & -0.6297 & -0.0524 & -0.7989 \\
\hline \multirow{3}{*}{ Quadratic 2} & Linear & 0.5067 & 1.7901 & 0.1686 \\
\hline & Quadratic & -1.0613 & -0.0533 & -0.7468 \\
\hline & Discrete & -1.0613 & -0.0533 & 1.2315 \\
\hline \multirow{3}{*}{ 3-Stepwise } & Linear & -0.4646 & 0.0167 & -0.6115 \\
\hline & Quadratic & -0.5011 & -0.0299 & -1.0587 \\
\hline & Discrete & -0.5011 & -0.0299 & -1.0029 \\
\hline
\end{tabular}

${ }^{a}$ Heterogeneity in $\omega$ is modelled through a MXL model

${ }^{\mathrm{b}} \omega$ is erroneously considered constant, so a MNL model is estimated

Bias in the estimated value of a hypothetical change in $X_{1}$ from the BAU level (6) to a level of 4 under each assumption about $\omega$ when the true Cost parameter presents scale heterogeneity (over 1,000 repetitions)

\begin{tabular}{|c|c|c|c|c|}
\hline \multirow{2}{*}{$\begin{array}{l}\text { True utility } \\
\text { specification }\end{array}$} & \multirow{2}{*}{$\begin{array}{l}\text { Assumed utility } \\
\text { specification }\end{array}$} & \multirow[b]{2}{*}{ Constant $^{b}$} & \multicolumn{2}{|c|}{ Mistaken heterogeneity source } \\
\hline & & & Lognormal $^{\mathrm{a}}$ & Triangular $^{\mathrm{a}}$ \\
\hline \multirow{3}{*}{ Linear } & Linear & -9.6028 & -0.7384 & -8.6848 \\
\hline & Quadratic & -9.6514 & -0.6068 & -52.7292 \\
\hline & Discrete & -9.6514 & -0.6064 & 1.8978 \\
\hline \multirow{3}{*}{ Quadratic 1} & Linear & -3.7311 & 1.0075 & -6.7809 \\
\hline & Quadratic & -5.8251 & -0.4843 & -10.8649 \\
\hline & Discrete & -5.8251 & -0.4846 & -7.3904 \\
\hline \multirow{3}{*}{ Quadratic 2} & Linear & 2.3437 & 8.2801 & 0.7799 \\
\hline & Quadratic & -4.9089 & -0.2466 & -3.4541 \\
\hline & Discrete & -4.9089 & -0.2466 & 5.6965 \\
\hline \multirow{3}{*}{ 3-Stepwise } & Linear & -8.5952 & 0.3093 & -11.3136 \\
\hline & Quadratic & -9.2704 & -0.5537 & -19.5887 \\
\hline & Discrete & -9.2704 & -0.5533 & -18.5557 \\
\hline
\end{tabular}

\footnotetext{
${ }^{\text {a }}$ Heterogeneity in $\omega$ is modelled through a MXL model

${ }^{\mathrm{b}} \omega$ is erroneously considered constant, so a MNL model is estimated
} 
MSE in the estimated value of a hypothetical change in $X_{1}$ from the BAU level (6) to a level of 4 under each assumption about $\omega$ when the true Cost parameter presents scale heterogeneity (over 1,000 repetitions)

\begin{tabular}{|c|c|c|c|c|}
\hline \multirow{2}{*}{$\begin{array}{l}\text { True utility } \\
\text { specification }\end{array}$} & \multirow{2}{*}{$\begin{array}{l}\text { Assumed utility } \\
\text { specification }\end{array}$} & \multirow[b]{2}{*}{ Constant $^{b}$} & \multicolumn{2}{|c|}{ Mistaken heterogeneity source } \\
\hline & & & Lognormal $^{\mathbf{a}}$ & Triangular $^{\mathrm{a}}$ \\
\hline \multirow{3}{*}{ Linear } & Linear & 92.241 & 1.285 & 41426.412 \\
\hline & Quadratic & 93.227 & 1.241 & 1258587.256 \\
\hline & Discrete & 93.227 & 1.241 & 158128.883 \\
\hline \multirow{3}{*}{ Quadratic 1} & Linear & 13.939 & 1.240 & 1783.032 \\
\hline & Quadratic & 34.021 & 0.507 & 12239.674 \\
\hline & Discrete & 34.022 & 0.507 & 2816.109 \\
\hline \multirow{3}{*}{ Quadratic 2} & Linear & 5.514 & 68.887 & 88413.094 \\
\hline & Quadratic & 24.224 & 0.299 & 262.178 \\
\hline & Discrete & 24.224 & 0.299 & 102677.237 \\
\hline \multirow{3}{*}{ 3-Stepwise } & Linear & 73.903 & 0.831 & 24584.229 \\
\hline & Quadratic & 86.020 & 1.103 & 4977.505 \\
\hline & Discrete & 86.021 & 1.103 & 44091.296 \\
\hline
\end{tabular}

${ }^{\text {a }}$ Heterogeneity in $\omega$ is modelled through a MXL model

${ }^{\mathrm{b}} \omega$ is erroneously considered constant, so a MNL model is estimated 
Relative bias in the estimated value of a change in $X_{1}$ from the BAU level (6) to a level of 2 under true, non-additive utility specifications (over 1,000 repetitions)

\begin{tabular}{llccc}
\hline True utility & Assumed utility & \multicolumn{3}{c}{ Relative bias } \\
\cline { 3 - 5 } specification & specification & $\mathbf{X}_{\mathbf{2}}=\mathbf{8}$ & $\mathbf{X}_{\mathbf{2}}=\mathbf{6}$ & $\mathbf{X}_{\mathbf{2}}=\mathbf{3}$ \\
\hline \multirow{3}{*}{ Cobb-Douglas 1 } & Linear & -0.0731 & -0.0182 & 0.1278 \\
& Quadratic & -0.0752 & -0.0205 & 0.1252 \\
& Discrete & -0.0752 & -0.0205 & 0.1252 \\
\hline \multirow{3}{*}{ Cobb-Douglas 2 } & Linear & -0.1271 & 0.0988 & 0.9131 \\
& Quadratic & -0.1196 & 0.1082 & 0.9294 \\
& Discrete & -0.1196 & 0.1082 & 0.9295 \\
\hline
\end{tabular}

Bias in the estimated value of a change in $X_{1}$ from the BAU level (6) to a level of 2 under true, non-additive utility specifications (over 1,000 repetitions)

\begin{tabular}{llccc}
\hline True utility & Assumed utility & \multicolumn{3}{c}{ Bias } \\
\cline { 3 - 5 } specification & specification & $\mathbf{X}_{\mathbf{2}}=\mathbf{8}$ & $\mathbf{X}_{\mathbf{2}}=\mathbf{6}$ & $\mathbf{X}_{\mathbf{2}}=\mathbf{3}$ \\
\hline \multirow{3}{*}{ Cobb-Douglas 1 } & Linear & -0.7128 & -0.1673 & 1.0249 \\
& Quadratic & -0.7340 & -0.1885 & 1.0037 \\
& Discrete & -0.7340 & -0.1885 & 1.0037 \\
\hline \multirow{3}{*}{ Cobb-Douglas 2 } & Linear & -0.4972 & 0.3068 & 1.6293 \\
& Quadratic & -0.4679 & 0.3361 & 1.6585 \\
& Discrete & -0.4679 & 0.3361 & 1.6585 \\
\hline
\end{tabular}

MSE in the estimated value of a hypothetical change in $X_{1}$ from the BAU level (6) to a level of 2 under true, non-additive utility specifications (over 1,000 repetitions)

\begin{tabular}{llccc}
\hline True utility & Assumed utility & \multicolumn{3}{c}{ MSE } \\
\cline { 3 - 5 } specification & specification & $\mathbf{X}_{\mathbf{2}}=\mathbf{8}$ & $\mathbf{X}_{\mathbf{2}}=\mathbf{6}$ & $\mathbf{X}_{\mathbf{2}}=\mathbf{3}$ \\
\hline \multirow{3}{*}{ Cobb-Douglas 1 } & Linear & 0.5275 & 0.0475 & 1.0698 \\
& Quadratic & 0.5593 & 0.0561 & 1.0279 \\
& Discrete & 0.5592 & 0.0561 & 1.0280 \\
\hline \multirow{3}{*}{ Cobb-Douglas 2 } & Linear & 0.2591 & 0.1061 & 2.6665 \\
& Quadratic & 0.2312 & 0.1252 & 2.7629 \\
& Discrete & 0.2312 & 0.1252 & 2.7630 \\
\hline
\end{tabular}


Relative bias in the estimated value of a hypothetical change in $X_{1}$ from the BAU level (6) to a level of 4 under true, non-additive utility specifications (over 1,000 repetitions)

\begin{tabular}{llccc}
\hline True utility & Assumed utility & \multicolumn{3}{c}{ Relative bias } \\
\cline { 3 - 5 } specification & specification & $\mathbf{X}_{\mathbf{2}}=\mathbf{8}$ & $\mathbf{X}_{\mathbf{2}}=\mathbf{6}$ & $\mathbf{X}_{\mathbf{2}}=\mathbf{3}$ \\
\hline \multirow{3}{*}{ Cobb-Douglas 1 } & Linear & -0.0217 & 0.0363 & 0.1904 \\
& Quadratic & -0.0041 & 0.0548 & 0.2117 \\
& Discrete & -0.0041 & 0.0548 & 0.2117 \\
\hline \multirow{3}{*}{ Cobb-Douglas 2 } & Linear & 0.1053 & 0.3913 & 1.4224 \\
& Quadratic & -0.0463 & 0.2004 & 1.0901 \\
& Discrete & -0.0464 & 0.2004 & 1.0901 \\
\hline
\end{tabular}

Bias in the estimated value of a hypothetical change in $X_{1}$ from the BAU level (6) to a level of 4 under true, non-additive utility specifications (over 1,000 repetitions)

\begin{tabular}{llccc}
\hline True utility & Assumed utility & \multicolumn{3}{c}{ Bias } \\
\cline { 3 - 5 } specification & specification & $\mathbf{X}_{\mathbf{2}}=\mathbf{8}$ & $\mathbf{X}_{\mathbf{2}}=\mathbf{6}$ & $\mathbf{X}_{\mathbf{2}}=\mathbf{3}$ \\
\hline \multirow{3}{*}{ Cobb-Douglas 1 } & Linear & -0.1002 & 0.1582 & 0.7230 \\
& Quadratic & -0.0191 & 0.2393 & 0.8041 \\
& Discrete & -0.0191 & 0.2393 & 0.8041 \\
\hline \multirow{3}{*}{ Cobb-Douglas 2 } & Linear & 0.1626 & 0.4800 & 1.0022 \\
& Quadratic & -0.0716 & 0.2459 & 0.7681 \\
& Discrete & -0.0716 & 0.2459 & 0.7681 \\
\hline
\end{tabular}

MSE in the estimated value of a hypothetical change in $X_{1}$ from the BAU level (6) to a level of 4 under true, non-additive utility specifications (over 1,000 repetitions)

\begin{tabular}{llccc}
\hline True utility & Assumed utility & \multicolumn{3}{c}{ MSE } \\
\cline { 3 - 5 } specification & specification & $\mathbf{X}_{\mathbf{2}}=\mathbf{8}$ & $\mathbf{X}_{\mathbf{2}}=\mathbf{6}$ & $\mathbf{X}_{\mathbf{2}}=\mathbf{3}$ \\
\hline \multirow{3}{*}{ Cobb-Douglas 1 } & Linear & 0.0149 & 0.0299 & 0.5276 \\
& Quadratic & 0.0171 & 0.0740 & 0.6633 \\
& Discrete & 0.0171 & 0.0740 & 0.6633 \\
\hline \multirow{3}{*}{ Cobb-Douglas 2 } & Linear & 0.0294 & 0.2334 & 1.0074 \\
& Quadratic & 0.0236 & 0.0790 & 0.6085 \\
& Discrete & 0.0237 & 0.0790 & 0.6085 \\
\hline
\end{tabular}


Relative bias in the estimated value of a of a hypothetical change in $\mathrm{X}_{1}$ from the BAU level (6) to a level of 2 under each assumption about $\omega$ when the true Cost parameter is lognormally-distributed and the true utility specification is non-additive (over 1,000 repetitions)"

\begin{tabular}{|c|c|c|c|c|}
\hline \multirow{2}{*}{$\begin{array}{l}\text { True utility } \\
\text { specification }\end{array}$} & \multirow{2}{*}{$\begin{array}{l}\text { Assumed } \\
\text { utility } \\
\text { specification }\end{array}$} & \multirow{2}{*}{$\begin{array}{c}\text { Correct } \\
\text { assumption } \\
\text { Lognormal }^{\mathbf{a}}\end{array}$} & \multicolumn{2}{|c|}{ 'Mistaken assumptions' } \\
\hline & & & Constant $^{\mathrm{b}}$ & Triangular $^{\mathrm{a}}$ \\
\hline \multirow{3}{*}{ Cobb-Douglas 1} & Linear & -0.0788 & -0.1005 & -0.0261 \\
\hline & Quadratic & -0.0750 & -0.1032 & -0.0182 \\
\hline & Discrete & -0.0750 & -0.1032 & -0.0182 \\
\hline \multirow{3}{*}{ Cobb-Douglas 2} & Linear & -0.1144 & -0.2763 & -0.0386 \\
\hline & Quadratic & -0.1054 & -0.2576 & 0.0321 \\
\hline & Discrete & -0.1054 & -0.2576 & 0.0310 \\
\hline
\end{tabular}

*Results for $\mathrm{X}_{2}=8$

${ }^{a}$ Heterogeneity in $\omega$ is modelled through a MXL model

${ }^{\mathrm{b}} \omega$ is erroneously considered constant, so a MNL model is estimated

Bias in the estimated value of a of a hypothetical change in $X_{1}$ from the BAU level (6) to a level of 2 under each assumption about $\omega$ when the true Cost parameter is lognormally-distributed and the true utility specification is non-additive (over 1,000 repetitions) ${ }^{*}$

\begin{tabular}{|c|c|c|c|c|}
\hline \multirow{2}{*}{$\begin{array}{l}\text { True utility } \\
\text { specification }\end{array}$} & \multirow{2}{*}{$\begin{array}{l}\text { Assumed utility } \\
\text { specification }\end{array}$} & \multirow{2}{*}{$\begin{array}{c}\text { Correct } \\
\text { assumption } \\
\text { Lognormal }^{\mathrm{a}}\end{array}$} & \multicolumn{2}{|c|}{ 'Mistaken assumptions' } \\
\hline & & & Constant $^{\mathrm{b}}$ & Triangular $^{\mathbf{a}}$ \\
\hline \multirow{3}{*}{ Cobb-Douglas 1} & Linear & -1.0280 & -1.3112 & -0.3409 \\
\hline & Quadratic & -0.9790 & -1.3466 & -0.2370 \\
\hline & Discrete & -0.9793 & -1.3466 & -0.2370 \\
\hline \multirow{3}{*}{ Cobb-Douglas 2} & Linear & -0.5987 & -1.4457 & -0.2022 \\
\hline & Quadratic & -0.5517 & -1.3479 & 0.1679 \\
\hline & Discrete & -0.5516 & -1.3478 & 0.1624 \\
\hline
\end{tabular}

${ }^{*}$ Results for $\mathrm{X}_{2}=8$

${ }^{\text {a }}$ Heterogeneity in $\omega$ is modelled through a MXL model

${ }^{\mathrm{b}} \omega$ is erroneously considered constant, so a MNL model is estimated 
MSE in the estimated value of a hypothetical change in $X_{1}$ from the BAU level (6) to a level of 2 when the true Cost parameter is lognormally-distributed and the true utility specification is non-additive (over 1,000 repetitions) ${ }^{*}$

\begin{tabular}{llccc}
\hline & & \multicolumn{4}{c}{$\begin{array}{c}\text { Correct } \\
\text { True utility }\end{array}$} & $\begin{array}{l}\text { Assumed utility } \\
\text { specification }\end{array}$ & specification & assumption & 'Mistaken assumptions' \\
\cline { 3 - 5 } & Lognormal $^{\text {a }}$ & Constant $^{\mathbf{b}}$ & Triangular $^{\mathbf{a}}$ \\
\hline \multirow{2}{*}{ Cobb-Douglas 1 } & Linear & 1.0974 & 1.7571 & 0.2212 \\
& Quadratic & 1.0008 & 1.8532 & 0.1650 \\
& Discrete & 1.0014 & 1.8533 & 0.1650 \\
\hline \multirow{3}{*}{ Cobb-Douglas 2 } & Linear & 0.3797 & 2.1208 & 139.5348 \\
& Quadratic & 0.3270 & 1.8486 & 6.8542 \\
& Discrete & 0.3269 & 1.8485 & 7.4081 \\
\hline
\end{tabular}

*Results for $\mathrm{X}_{2}=8$

${ }^{\text {a }}$ Heterogeneity in $\omega$ is modelled through a MXL model

${ }^{\mathrm{b}} \omega$ is erroneously considered constant, so a MNL model is estimated

Relative bias in the estimated value of a hypothetical change in $X_{1}$ from the BAU level (6) to a level of 4 when the true Cost parameter is lognormally-distributed and the true utility specification is non-additive (over 1,000 repetitions)*

\begin{tabular}{|c|c|c|c|c|}
\hline \multirow{2}{*}{$\begin{array}{l}\text { True utility } \\
\text { specification }\end{array}$} & \multirow{2}{*}{$\begin{array}{l}\text { Assumed utility } \\
\text { specification }\end{array}$} & $\begin{array}{c}\text { Correct } \\
\text { assumption }\end{array}$ & \multicolumn{2}{|c|}{ 'Mistaken assumptions' } \\
\hline & & Lognormal $^{\text {a }}$ & Constant $^{\mathrm{b}}$ & Triangular $^{\mathrm{a}}$ \\
\hline \multirow{3}{*}{ Cobb-Douglas 1} & Linear & -0.0277 & -0.0506 & 0.0279 \\
\hline & Quadratic & -0.0449 & -0.0356 & -0.0096 \\
\hline & Discrete & -0.0449 & -0.0356 & -0.0096 \\
\hline \multirow{3}{*}{ Cobb-Douglas 2} & Linear & 0.1214 & -0.0836 & 0.2173 \\
\hline & Quadratic & -0.0548 & -0.4990 & 0.1033 \\
\hline & Discrete & -0.0548 & -0.4990 & 0.1022 \\
\hline
\end{tabular}

*Results for $\mathrm{X}_{2}=8$

${ }^{a}$ Heterogeneity in $\omega$ is modelled through a MXL model

${ }^{\mathrm{b}} \omega$ is erroneously considered constant, so a MNL model is estimated 
Bias in the estimated value of a hypothetical change in $X_{1}$ from the BAU level (6) to a level of 4 when the true Cost parameter is lognormally-distributed and the true utility specification is non-additive (over 1,000 repetitions) ${ }^{*}$

\begin{tabular}{llccc}
\hline & & \multicolumn{4}{c}{$\begin{array}{c}\text { Correct } \\
\text { True utility }\end{array}$} & $\begin{array}{l}\text { Assumed utility } \\
\text { specification }\end{array}$ & specification & assumption & 'Mistaken assumptions' \\
\cline { 3 - 5 } & Lognormal $^{\text {a }}$ & Constant $^{\mathbf{b}}$ & Triangular $^{\text {' }}$ \\
\hline \multirow{2}{*}{ Cobb-Douglas 1 } & Linear & -0.1712 & -0.3127 & 0.1724 \\
& Quadratic & -0.2777 & -0.2201 & -0.0594 \\
& Discrete & -0.2778 & -0.2201 & -0.0593 \\
\hline \multirow{3}{*}{ Cobb-Douglas 2 } & Linear & 0.2507 & -0.1727 & 0.4490 \\
& Quadratic & -0.1132 & -1.0311 & 0.2135 \\
& Discrete & -0.1132 & -1.0310 & 0.2113 \\
\hline
\end{tabular}

*Results for $\mathrm{X}_{2}=8$

${ }^{\text {a }}$ Heterogeneity in $\omega$ is modelled through a MXL model

${ }^{\mathrm{b}} \omega$ is erroneously considered constant, so a MNL model is estimated

MSE in the estimated value of a hypothetical change in $X_{1}$ from the BAU level (6) to a level of 4 when the true Cost parameter is lognormally-distributed and the true utility specification is non-additive (over 1,000 repetitions) ${ }^{*}$

\begin{tabular}{|c|c|c|c|c|}
\hline \multirow{2}{*}{$\begin{array}{l}\text { True utility } \\
\text { specification }\end{array}$} & \multirow{2}{*}{$\begin{array}{l}\text { Assumed utility } \\
\text { specification }\end{array}$} & \multirow{2}{*}{$\begin{array}{c}\text { Correct } \\
\text { assumption } \\
\text { Lognormal }^{\mathbf{a}}\end{array}$} & \multicolumn{2}{|c|}{ 'Mistaken assumptions' } \\
\hline & & & Constant $^{b}$ & Triangular $^{\mathrm{a}}$ \\
\hline \multirow{3}{*}{ Cobb-Douglas 1} & Linear & 0.0395 & 0.1073 & 0.0560 \\
\hline & Quadratic & 0.1036 & 0.0774 & 0.0476 \\
\hline & Discrete & 0.1037 & 0.0774 & 0.0476 \\
\hline \multirow{3}{*}{ Cobb-Douglas 2} & Linear & 0.0682 & 0.0376 & 35.0751 \\
\hline & Quadratic & 0.0449 & 1.0953 & 1.2251 \\
\hline & Discrete & 0.0449 & 1.0952 & 1.3163 \\
\hline
\end{tabular}

*Results for $\mathrm{X}_{2}=8$

${ }^{\text {a }}$ Heterogeneity in $\omega$ is modelled through a MXL model

${ }^{\mathrm{b}} \omega$ is erroneously considered constant, so a MNL model is estimated 
Relative bias in the estimated value of a hypothetical change in $\mathrm{X}_{1}$ from the BAU level (6) to a level of 2 when the true Cost parameter is lognormally-distributed and the true utility specification is non-additive (over 1,000 repetitions) ${ }^{*}$

\begin{tabular}{llccc}
\hline & & \multicolumn{4}{c}{$\begin{array}{c}\text { Correct } \\
\text { True utility } \\
\text { specification }\end{array}$} & $\begin{array}{l}\text { Assumed utility } \\
\text { specification }\end{array}$ & \begin{tabular}{cccc} 
assumption \\
\cline { 3 - 5 }
\end{tabular} & $\begin{array}{l}\text { 'Mognormal } \\
\text { ' }\end{array}$ & Constant $^{\text {b }}$ & Triangular $^{\text {a }}$ \\
\hline \multirow{2}{*}{ Cobb-Douglas 1 } & Quadratic & -0.0242 & -0.0472 & 0.0316 \\
& Discrete & -0.0202 & -0.0501 & 0.0440 \\
& Linear & -0.0202 & -0.0501 & 0.0400 \\
\hline \multirow{3}{*}{ Cobb-Douglas 2 } & Quadratic & 0.1148 & -0.0890 & 0.2102 \\
& Discrete & 0.1261 & -0.0655 & 0.2992 \\
& & 0.1261 & -0.0655 & 0.2978 \\
\hline
\end{tabular}

*Results for $\mathrm{X}_{2}=6$

${ }^{\text {a }}$ Heterogeneity in $\omega$ is modelled through a MXL model

${ }^{\mathrm{b}} \omega$ is erroneously considered constant, so a MNL model is estimated

Bias in the estimated value of a hypothetical change in $X_{1}$ from the BAU level (6) to a level of 2 when the true Cost parameter is lognormally-distributed and the true utility specification is non-additive (over 1,000 repetitions) ${ }^{*}$

\begin{tabular}{llccc}
\hline & & \multicolumn{4}{c}{$\begin{array}{c}\text { Correct } \\
\text { True utility }\end{array}$} & $\begin{array}{l}\text { Assumed utility } \\
\text { specification }\end{array}$ & specification & assumption & 'Mistaken assumptions' \\
\cline { 3 - 5 } & Lognormal $^{\mathbf{a}}$ & Constant $^{\mathbf{b}}$ & Triangular $^{\mathbf{a}}$ \\
\hline \multirow{2}{*}{ Cobb-Douglas 1 } & Linear & -0.2982 & -0.5813 & 0.3889 \\
& Quadratic & -0.2492 & -0.6108 & 0.4928 \\
& Discrete & -0.2495 & -0.6108 & 0.4928 \\
\hline \multirow{3}{*}{ Cobb-Douglas 2 } & Linear & 0.4770 & -0.3699 & 0.8736 \\
& Quadratic & 0.5241 & -0.2721 & 1.2436 \\
& Discrete & 0.5241 & -0.2721 & 1.2381 \\
\hline
\end{tabular}

*Results for $\mathrm{X}_{2}=6$

${ }^{a}$ Heterogeneity in $\omega$ is modelled through a MXL model

${ }^{\mathrm{b}} \omega$ is erroneously considered constant, so a MNL model is estimated 
MSE in the estimated value of a hypothetical change in $X_{1}$ from the BAU level (6) to a level of 2 when the true Cost parameter is lognormally-distributed and the true utility specification is non-additive (over 1,000 repetitions) ${ }^{*}$

\begin{tabular}{|c|c|c|c|c|}
\hline \multirow{2}{*}{$\begin{array}{l}\text { True utility } \\
\text { specification }\end{array}$} & \multirow{2}{*}{$\begin{array}{l}\text { Assumed utility } \\
\text { specification }\end{array}$} & $\begin{array}{c}\text { Correct } \\
\text { assumption }\end{array}$ & \multicolumn{2}{|c|}{ 'Mistaken assumptions' } \\
\hline & & Lognormal $^{\mathrm{a}}$ & Constant $^{\mathrm{b}}$ & Triangular $^{\mathrm{a}}$ \\
\hline \multirow{3}{*}{ Cobb-Douglas 1} & Linear & 0.1296 & 0.3759 & 0.2562 \\
\hline & Quadratic & 0.1044 & 0.4203 & 0.3516 \\
\hline & Discrete & 0.1045 & 0.4203 & 0.3517 \\
\hline \multirow{3}{*}{ Cobb-Douglas 2} & Linear & 0.2488 & 0.1677 & 140.2571 \\
\hline & Quadratic & 0.2973 & 0.1059 & 8.3726 \\
\hline & Discrete & 0.2973 & 0.1059 & 8.9147 \\
\hline
\end{tabular}

Relative bias in the estimated value of a hypothetical change in $X_{1}$ from the BAU level (6) to a level of 4 when the true Cost parameter is lognormally-distributed and the true utility specification is non-additive (over 1,000 repetitions)*

\begin{tabular}{llccc}
\hline & & \multicolumn{4}{c}{$\begin{array}{c}\text { Correct } \\
\text { True utility } \\
\text { specification }\end{array}$} & $\begin{array}{l}\text { Assumed utility } \\
\text { specification }\end{array}$ & \begin{tabular}{cccc} 
assumption \\
\cline { 3 - 5 }
\end{tabular} & 'Mistaken assumptions' \\
\hline \multirow{2}{*}{ Cobb-Douglas 1 1 } & 'inear & 0.0299 & 0.0057 & 0.0888 \\
& Quadratic & 0.0117 & 0.0215 & 0.0491 \\
& Discrete & 0.0116 & 0.0215 & 0.0491 \\
\hline \multirow{2}{*}{ Cobb-Douglas 2 } & Linear & 0.4115 & 0.1536 & 0.5323 \\
& Quadratic & 0.1898 & -0.3694 & 0.3889 \\
& Discrete & 0.1898 & -0.3693 & 0.3875 \\
\hline
\end{tabular}

*Results for $\mathrm{X}_{2}=6$

${ }^{\text {a }}$ Heterogeneity in $\omega$ is modelled through a MXL model

${ }^{\mathrm{b}} \omega$ is erroneously considered constant, so a MNL model is estimated 
Bias in the estimated value of a hypothetical change in $X_{1}$ from the BAU level (6) to a level of 4 when the true Cost parameter is lognormally-distributed and the true utility specification is non-additive (over 1,000 repetitions) ${ }^{*}$

\begin{tabular}{llccc}
\hline \multirow{2}{*}{$\begin{array}{l}\text { True utility } \\
\text { specification }\end{array}$} & $\begin{array}{l}\text { Assumed utility } \\
\text { specification }\end{array}$ & $\begin{array}{c}\text { Correct } \\
\text { assumption }\end{array}$ & 'Mistaken assumptions' \\
\cline { 3 - 5 } & Lognormal & ' & Constant $^{\text {' }}$ & Triangular $^{\text {' }}$ \\
\hline \multirow{2}{*}{ Cobb-Douglas 1 } & Linear & 0.1746 & 0.0330 & 0.5181 \\
& Quadratic & 0.0681 & 0.1257 & 0.2864 \\
& Discrete & 0.0680 & 0.1257 & 0.2864 \\
\hline \multirow{3}{*}{ Cobb-Douglas 2 } & Linear & 0.6755 & 0.2521 & 0.8738 \\
& Quadratic & 0.3116 & -0.6063 & 0.6383 \\
& Discrete & 0.3116 & -0.6063 & 0.6361 \\
\hline
\end{tabular}

"Results for $\mathrm{X}_{2}=6$

${ }^{\text {a }}$ Heterogeneity in $\omega$ is modelled through a MXL model

${ }^{\mathrm{b}} \omega$ is erroneously considered constant, so a MNL model is estimated

MSE in the estimated value of a hypothetical change in $X_{1}$ from the BAU level (6) to a level of 4 when the true Cost parameter is lognormally-distributed and the true utility specification is non-additive (over 1,000 repetitions) *

\begin{tabular}{llccc}
\hline & & \multicolumn{4}{c}{$\begin{array}{c}\text { Correct } \\
\text { True utility }\end{array}$} & $\begin{array}{l}\text { Assumed utility } \\
\text { specification }\end{array}$ & specification & assumption & 'Mistaken assumptions' \\
\cline { 3 - 5 } & Lognormal $^{\mathbf{a}}$ & Constant $^{\mathbf{b}}$ & Triangular $^{\mathbf{a}}$ \\
\hline \multirow{2}{*}{ Cobb-Douglas 1 } & Linear & 0.0406 & 0.0106 & 0.2947 \\
& Quadratic & 0.0312 & 0.0448 & 0.1261 \\
& Discrete & 0.0312 & 0.0448 & 0.1261 \\
\hline \multirow{3}{*}{ Cobb-Douglas 2 } & Linear & 0.4617 & 0.0713 & 35.6370 \\
& Quadratic & 0.1292 & 0.3998 & 1.5869 \\
& Discrete & 0.1292 & 0.3997 & 1.6763 \\
\hline
\end{tabular}

*Results for $\mathrm{X}_{2}=6$

${ }^{\text {a }}$ Heterogeneity in $\omega$ is modelled through a MXL model

${ }^{\mathrm{b}} \omega$ is erroneously considered constant, so a MNL model is estimated 
Relative bias in the estimated value of a hypothetical change in $X_{1}$ from the BAU level (6) to a level of 2 when the true Cost parameter is lognormally-distributed and the true utility specification is non-additive (over 1,000 repetitions) ${ }^{*}$

\begin{tabular}{|c|c|c|c|c|}
\hline \multirow{2}{*}{$\begin{array}{l}\text { True utility } \\
\text { specification }\end{array}$} & \multirow{2}{*}{$\begin{array}{l}\text { Assumed utility } \\
\text { specification }\end{array}$} & \multirow{2}{*}{$\begin{array}{c}\text { Correct } \\
\text { assumption } \\
\text { Lognormal }^{\text {a }}\end{array}$} & \multicolumn{2}{|c|}{ 'Mistaken assumptions' } \\
\hline & & & Constant $^{\mathrm{b}}$ & Triangular $^{\mathbf{a}}$ \\
\hline \multirow{3}{*}{ Cobb-Douglas 1} & Linear & 0.1209 & 0.0945 & 0.1849 \\
\hline & Quadratic & 0.1255 & 0.0912 & 0.1946 \\
\hline & Discrete & 0.1254 & 0.0912 & 0.1946 \\
\hline \multirow{3}{*}{ Cobb-Douglas 2} & Linear & 0.9409 & 0.5862 & 1.1070 \\
\hline & Quadratic & 0.9606 & 0.6271 & 1.2620 \\
\hline & Discrete & 0.9606 & 0.6271 & 1.2597 \\
\hline
\end{tabular}

"Results for $\mathrm{X}_{2}=3$

${ }^{\text {a }}$ Heterogeneity in $\omega$ is modelled through a MXL model

${ }^{\mathrm{b}} \omega$ is erroneously considered constant, so a MNL model is estimated

Bias in the estimated value of a hypothetical change in $X_{1}$ from the BAU level (6) to a level of 2 when the true Cost parameter is lognormally-distributed and the true utility specification is non-additive (over 1,000 repetitions) ${ }^{*}$

\begin{tabular}{llccc}
\hline \multirow{2}{*}{$\begin{array}{l}\text { True utility } \\
\text { specification }\end{array}$} & $\begin{array}{l}\text { Assumed utility } \\
\text { specification }^{*}\end{array}$ & $\begin{array}{c}\text { Correct } \\
\text { assumption }\end{array}$ & 'Mistaken assumptions' \\
\cline { 3 - 5 } Cobb-Douglas 1 & Linear & 1.2970 & 1.0139 & 1.9841 \\
& Quadratic & 1.3460 & 0.9784 & 2.0880 \\
& Discrete & 1.3457 & 0.9784 & 2.0880 \\
\hline \multirow{2}{*}{ Cobb-Douglas 2 } & Linear & 2.2465 & 1.3995 & 2.6430 \\
& Quadratic & 2.2935 & 1.4973 & 3.0131 \\
& Discrete & 2.2935 & 1.4973 & 3.0075 \\
\hline
\end{tabular}

*Results for $\mathrm{X}_{2}=3$

${ }^{\text {a }}$ Heterogeneity in $\omega$ is modelled through a MXL model

${ }^{\mathrm{b}} \omega$ is erroneously considered constant, so a MNL model is estimated 
MSE in the estimated value of a hypothetical change in $X_{1}$ from the BAU level (6) to a level of 2 when the true Cost parameter is lognormally-distributed and the true utility specification is non-additive (over 1,000 repetitions) ${ }^{*}$

\begin{tabular}{|c|c|c|c|c|}
\hline \multirow{2}{*}{$\begin{array}{l}\text { True utility } \\
\text { specification }\end{array}$} & \multirow{2}{*}{$\begin{array}{l}\text { Assumed utility } \\
\text { specification }\end{array}$} & \multirow{2}{*}{$\begin{array}{c}\text { Correct } \\
\text { assumption } \\
\text { Lognormal }^{\mathrm{a}}\end{array}$} & \multicolumn{2}{|c|}{ 'Mistaken assumptions' } \\
\hline & & & Constant $^{\mathrm{b}}$ & Triangular $^{\mathbf{a}}$ \\
\hline \multirow{3}{*}{ Cobb-Douglas 1} & Linear & 1.7229 & 1.0659 & 4.0416 \\
\hline & Quadratic & 1.8540 & 0.9971 & 4.4685 \\
\hline & Discrete & 1.8532 & 0.9971 & 4.4685 \\
\hline \multirow{3}{*}{ Cobb-Douglas 2} & Linear & 5.0678 & 1.9895 & 146.4794 \\
\hline & Quadratic & 5.2828 & 2.2738 & 15.9045 \\
\hline & Discrete & 5.2829 & 2.2739 & 16.4269 \\
\hline
\end{tabular}

"Results for $\mathrm{X}_{2}=3$

${ }^{\text {a }}$ Heterogeneity in $\omega$ is modelled through a MXL model

${ }^{\mathrm{b}} \omega$ is erroneously considered constant, so a MNL model is estimated

Relative bias in the estimated value of a hypothetical change in $X_{1}$ from the BAU level (6) to a level of 4 when the true Cost parameter is lognormally-distributed and the true utility specification is non-additive (over 1,000 repetitions)*

\begin{tabular}{llccc}
\hline & & \multicolumn{4}{c}{$\begin{array}{c}\text { Correct } \\
\text { True utility } \\
\text { specification }\end{array}$} & $\begin{array}{l}\text { Assumed utility } \\
\text { specification }\end{array}$ & \begin{tabular}{ccc} 
assumption \\
\cline { 3 - 5 }
\end{tabular} & 'Mognormal $^{\mathbf{a}}$ & Constant $^{\mathbf{b}}$ & Triangular $^{\mathbf{a}}$ \\
\hline \multirow{3}{*}{ Cobb-Douglas 1 1 } & Linear & 0.1831 & 0.1552 & 0.2506 \\
& Quadratic & 0.1621 & 0.1734 & 0.2051 \\
& Discrete & 0.1621 & 0.1734 & 0.2051 \\
\hline \multirow{3}{*}{ Cobb-Douglas 2 } & Linear & 1.4576 & 1.0085 & 1.6679 \\
& Quadratic & 1.0716 & 0.0980 & 1.4181 \\
& Discrete & 1.0716 & 0.0981 & 1.4158 \\
\hline
\end{tabular}

*Results for $\mathrm{X}_{2}=3$

${ }^{\text {a }}$ Heterogeneity in $\omega$ is modelled through a MXL model

${ }^{\mathrm{b}} \omega$ is erroneously considered constant, so a MNL model is estimated 
Bias in the estimated value of a hypothetical change in $X_{1}$ from the BAU level (6) to a level of 4 when the true Cost parameter is lognormally-distributed and the true utility specification is non-additive (over 1,000 repetitions) ${ }^{*}$

\begin{tabular}{llccc}
\hline & & \multicolumn{4}{c}{$\begin{array}{c}\text { Correct } \\
\text { True utility }\end{array}$} & $\begin{array}{l}\text { Assumed utility } \\
\text { specification }\end{array}$ & specification & \begin{tabular}{ccc} 
assumption \\
\cline { 3 - 5 }
\end{tabular} & 'Mognormal $^{\text {a }}$ & Constant $^{\text {b }}$ & Triangular $^{\text {a }}$ \\
\hline \multirow{2}{*}{ Cobb-Douglas 1 } & Linear & 0.9303 & 0.7887 & 1.2738 \\
& Quadratic & 0.8238 & 0.8814 & 1.0421 \\
& Discrete & 0.8237 & 0.8814 & 1.0421 \\
\hline \multirow{3}{*}{ Cobb-Douglas 2 } & Linear & 1.3742 & 0.9507 & 1.5725 \\
& Quadratic & 1.0103 & 0.0924 & 1.3370 \\
& Discrete & 1.0103 & 0.0924 & 1.3347 \\
\hline
\end{tabular}

*Results for $\mathrm{X}_{2}=3$

${ }^{\text {a }}$ Heterogeneity in $\omega$ is modelled through a MXL model

${ }^{\mathrm{b}} \omega$ is erroneously considered constant, so a MNL model is estimated

MSE in the estimated value of a hypothetical change in $X_{1}$ from the BAU level (6) to a level of 4 when the true Cost parameter is lognormally-distributed and the true utility specification is non-additive (over 1,000 repetitions)*

\begin{tabular}{llccc}
\hline & & \multicolumn{4}{c}{$\begin{array}{c}\text { Correct } \\
\text { True utility }\end{array}$} & $\begin{array}{l}\text { Assumed utility } \\
\text { specification }\end{array}$ & specification & assumption & 'Mistaken assumptions' \\
\cline { 3 - 5 } & Lognormal $^{\mathbf{a}}$ & Constant $^{\mathbf{b}}$ & Triangular $^{\text {a }}$ \\
\hline \multirow{2}{*}{ Cobb-Douglas 1 } & Linear & 0.8756 & 0.6316 & 1.6489 \\
& Quadratic & 0.7051 & 0.8058 & 1.1300 \\
& Discrete & 0.7050 & 0.8058 & 1.1301 \\
\hline \multirow{3}{*}{ Cobb-Douglas 2 } & Linear & 1.8938 & 0.9117 & 37.3462 \\
& Quadratic & 1.0527 & 0.0407 & 2.9670 \\
& Discrete & 1.0528 & 0.0407 & 3.0533 \\
\hline
\end{tabular}

*Results for $\mathrm{X}_{2}=3$

${ }^{\text {a }}$ Heterogeneity in $\omega$ is modelled through a MXL model

b $\omega$ is erroneously considered constant, so a MNL model is estimated 
Relative bias in the estimated value of a hypothetical change in $X_{1}$ from the BAU level (6) to a level of 2 under each assumption about $\omega$ when the true Cost parameter presents scale heterogeneity and the true utility specification is nonadditive (over 1,000 repetitions) ${ }^{*}$

\begin{tabular}{llccc}
\hline True utility & Assumed utility & & \multicolumn{2}{c}{ Mistaken heterogeneity source $^{\mathbf{2}}$ specification } \\
\cline { 4 - 5 } & Specification & Constant $^{\mathbf{b}}$ & Lognormal $^{\mathbf{a}}$ & Triangular $^{\mathbf{a}}$ \\
\hline \multirow{2}{*}{ Cobb-Douglas 1 } & Linear & -0.4722 & -0.0865 & -0.6127 \\
& Quadratic & -0.4688 & -0.0790 & -0.6706 \\
& Discrete & -0.4688 & -0.0790 & -0.9674 \\
\hline \multirow{3}{*}{ Cobb-Douglas 2 } & Linear & -0.7725 & -0.1098 & -0.7988 \\
& Quadratic & -0.7484 & -0.0952 & -0.7428 \\
& Discrete & -0.7484 & -0.0952 & -0.4184 \\
\hline
\end{tabular}

*Results for $\mathrm{X}_{2}=8$

${ }^{a}$ Heterogeneity in $\omega$ is modelled through a MXL model

${ }^{\mathrm{b}} \omega$ is erroneously considered constant, so a MNL model is estimated

Bias in the estimated value of a hypothetical change in $X_{1}$ from the BAU level (6) to a level of 2 under each assumption about $\omega$ when the true Cost parameter presents scale heterogeneity and the true utility specification is non-additive (over 1,000 repetitions)

\begin{tabular}{|c|c|c|c|c|}
\hline \multirow{2}{*}{$\begin{array}{l}\text { True utility } \\
\text { specification }\end{array}$} & \multirow{2}{*}{$\begin{array}{l}\text { Assumed utility } \\
\text { specification }\end{array}$} & \multirow[b]{2}{*}{ Constant $^{b}$} & \multicolumn{2}{|c|}{ Mistaken heterogeneity source } \\
\hline & & & Lognormal $^{\mathrm{a}}$ & Triangular $^{\mathbf{a}}$ \\
\hline \multirow{3}{*}{ Cobb-Douglas 1} & Linear & -17.0461 & -3.1221 & -22.1160 \\
\hline & Quadratic & -16.9214 & -2.8534 & -24.2089 \\
\hline & Discrete & -16.9214 & -2.8534 & -34.9233 \\
\hline \multirow{3}{*}{ Cobb-Douglas 2} & Linear & -11.1796 & -1.5884 & -11.5593 \\
\hline & Quadratic & -10.8309 & -1.3782 & -10.7497 \\
\hline & Discrete & -10.8308 & -1.3774 & -6.0553 \\
\hline
\end{tabular}

${ }^{*}$ Results for $\mathrm{X}_{2}=8$

${ }^{\text {a }}$ Heterogeneity in $\omega$ is modelled through a MXL model

${ }^{\mathrm{b}} \omega$ is erroneously considered constant, so a MNL model is estimated 
MSE in the estimated value of a hypothetical change in $X_{1}$ from the BAU level (6) to a level of 2 under each assumption about $\omega$ when the true Cost parameter presents scale heterogeneity and the true utility specification is non-additive (over 1,000 repetitions)

\begin{tabular}{|c|c|c|c|c|}
\hline \multirow{2}{*}{$\begin{array}{l}\text { True utility } \\
\text { specification }\end{array}$} & \multirow{2}{*}{$\begin{array}{l}\text { Assumed utility } \\
\text { specification }\end{array}$} & \multirow[b]{2}{*}{ Constant $^{b}$} & \multicolumn{2}{|c|}{ Mistaken heterogeneity source } \\
\hline & & & Lognormal $^{\mathbf{a}}$ & Triangular $^{\mathrm{a}}$ \\
\hline \multirow{3}{*}{ Cobb-Douglas 1} & Linear & 290.675 & 11.747 & 29820.553 \\
\hline & Quadratic & 286.437 & 10.222 & 52803.643 \\
\hline & Discrete & 286.437 & 10.222 & 7894.947 \\
\hline \multirow{3}{*}{ Cobb-Douglas 2} & Linear & 125.048 & 3.100 & 2011.415 \\
\hline & Quadratic & 117.377 & 2.501 & 823.753 \\
\hline & Discrete & 117.376 & 2.499 & 3224.095 \\
\hline
\end{tabular}

*Results for $\mathrm{X}_{2}=8$

${ }^{\text {a }}$ Heterogeneity in $\omega$ is modelled through a MXL model

${ }^{\mathrm{b}} \omega$ is erroneously considered constant, so a MNL model is estimated

Relative bias in the estimated value of a hypothetical change in $X_{1}$ from the BAU level (6) to a level of 4 under each assumption about $\omega$ when the true Cost parameter presents scale heterogeneity and the true utility specification is nonadditive (over 1,000 repetitions) ${ }^{*}$

\begin{tabular}{|c|c|c|c|c|}
\hline \multirow{2}{*}{$\begin{array}{l}\text { True utility } \\
\text { specification }\end{array}$} & \multirow{2}{*}{$\begin{array}{l}\text { Assumed utility } \\
\text { specification }\end{array}$} & \multirow[b]{2}{*}{ Constant $^{\mathrm{b}}$} & \multicolumn{2}{|c|}{ Mistaken heterogeneity source } \\
\hline & & & Lognormal $^{\mathbf{a}}$ & Triangular $^{\mathbf{a}}$ \\
\hline \multirow{3}{*}{ Cobb-Douglas 1} & Linear & -0.4429 & -0.0358 & -0.5912 \\
\hline & Quadratic & -0.4974 & -0.0786 & -0.6450 \\
\hline & Discrete & -0.4974 & -0.0786 & -0.9634 \\
\hline \multirow{3}{*}{ Cobb-Douglas 2} & Linear & -0.7120 & 0.1272 & -0.7452 \\
\hline & Quadratic & -1.0322 & -0.1363 & -0.7595 \\
\hline & Discrete & -1.0322 & -0.1361 & -0.4341 \\
\hline
\end{tabular}

*Results for $\mathrm{X}_{2}=8$

${ }^{\text {a }}$ Heterogeneity in $\omega$ is modelled through a MXL model

b $\omega$ is erroneously considered constant, so a MNL model is estimated 
Bias in the estimated value of a hypothetical change in $X_{1}$ from the BAU level (6) to a level of 4 under each assumption about $\omega$ when the true Cost parameter presents scale heterogeneity and the true utility specification is non-additive (over 1,000 repetitions) ${ }^{*}$

\begin{tabular}{|c|c|c|c|c|}
\hline \multirow{2}{*}{$\begin{array}{l}\text { True utility } \\
\text { specification }\end{array}$} & \multirow{2}{*}{$\begin{array}{l}\text { Assumed utility } \\
\text { specification }\end{array}$} & \multirow[b]{2}{*}{ Constant $^{b}$} & \multicolumn{2}{|c|}{ Mistaken heterogeneity source } \\
\hline & & & Lognormal $^{\mathbf{a}}$ & Triangular ${ }^{\mathrm{a}}$ \\
\hline \multirow{3}{*}{ Cobb-Douglas 1} & Linear & -7.5749 & -0.6129 & -10.1098 \\
\hline & Quadratic & -8.5055 & -1.3448 & -11.0297 \\
\hline & Discrete & -8.5055 & -1.3447 & -16.4744 \\
\hline \multirow{3}{*}{ Cobb-Douglas 2} & Linear & -4.0685 & 0.7272 & -4.2583 \\
\hline & Quadratic & -5.8985 & -0.7786 & -4.3404 \\
\hline & Discrete & -5.8985 & -0.7780 & -2.4807 \\
\hline
\end{tabular}

"Results for $\mathrm{X}_{2}=8$

${ }^{\text {a }}$ Heterogeneity in $\omega$ is modelled through a MXL model

${ }^{\mathrm{b}} \omega$ is erroneously considered constant, so a MNL model is estimated

MSE in the estimated value of a hypothetical change in $X_{1}$ from the BAU level (6) to a level of 4 under each assumption about $\omega$ when the true Cost parameter presents scale heterogeneity and the true utility specification is non-additive (over 1,000 repetitions)

\begin{tabular}{llccc}
\hline True utility & $\begin{array}{l}\text { Assumed utility } \\
\text { specification }\end{array}$ & & \multicolumn{2}{c}{ Mistaken heterogeneity source $^{\mathbf{s}}$} \\
\cline { 4 - 5 } & Specification & Constant $^{\mathbf{b}}$ & Lognormal $^{\mathbf{a}}$ & Triangular $^{\mathbf{a}}$ \\
\hline \multirow{2}{*}{ Cobb-Douglas 1 1 } & Linear & 57.405 & 0.875 & 7435.068 \\
& Quadratic & 72.431 & 2.363 & 13664.030 \\
& Discrete & 72.431 & 2.362 & 1904.534 \\
\hline \multirow{2}{*}{ Cobb-Douglas 2 } & Linear & 16.569 & 0.673 & 487.583 \\
& Quadratic & 34.849 & 0.975 & 118.267 \\
& Discrete & 34.849 & 0.974 & 382.930 \\
\hline
\end{tabular}

*Results for $\mathrm{X}_{2}=8$

${ }^{\text {a }}$ Heterogeneity in $\omega$ is modelled through a MXL model

${ }^{\mathrm{b}} \omega$ is erroneously considered constant, so a MNL model is estimated 
Relative bias in the estimated value of a hypothetical change in $X_{1}$ from the BAU level (6) to a level of 2 under each assumption about $\omega$ when the true Cost parameter presents scale heterogeneity and the true utility specification is nonadditive (over 1,000 repetitions)*

\begin{tabular}{llccc}
\hline True utility & Assumed utility & & \multicolumn{2}{c}{ Mistaken heterogeneity source $^{\mathbf{2}}$ specification } \\
\cline { 4 - 5 } & Specification & Constant $^{\mathbf{b}}$ & Lognormal $^{\mathbf{a}}$ & Triangular $^{\mathbf{a}}$ \\
\hline \multirow{2}{*}{ Cobb-Douglas 1 } & Linear & -0.4410 & -0.0324 & -0.5897 \\
& Quadratic & -0.4373 & -0.0245 & -0.6511 \\
& Discrete & -0.4373 & -0.0245 & -0.9655 \\
\hline \multirow{3}{*}{ Cobb-Douglas 2 } & Linear & -0.7137 & 0.1206 & -0.7467 \\
& Quadratic & -0.6833 & 0.1389 & -0.6763 \\
& Discrete & -0.6833 & 0.1390 & -0.2679 \\
\hline
\end{tabular}

*Results for $\mathrm{X}_{2}=6$

${ }^{\text {a }}$ Heterogeneity in $\omega$ is modelled through a MXL model

${ }^{\mathrm{b}} \omega$ is erroneously considered constant, so a MNL model is estimated

Bias in the estimated value of a hypothetical change in $X_{1}$ from the BAU level (6) to a level of 2 under each assumption about $\omega$ when the true Cost parameter presents scale heterogeneity and the true utility specification is non-additive (over 1,000 repetitions) ${ }^{*}$

\begin{tabular}{llccc}
\hline True utility & Assumed utility & & \multicolumn{2}{c}{ Mistaken heterogeneity source $^{\text {Specification }}$} \\
\cline { 5 - 5 } & specification & Constant $^{\mathbf{b}}$ & Lognormal $^{\mathbf{a}}$ & Triangular $^{\mathbf{a}}$ \\
\hline \multirow{3}{*}{ Cobb-Douglas 1 } & Linear & -15.0278 & -1.1037 & -20.0977 \\
& Quadratic & -14.9030 & -0.8351 & -22.1905 \\
& Discrete & -14.9030 & -0.8351 & -32.9049 \\
\hline \multirow{3}{*}{ Cobb-Douglas 2 } & Linear & -8.2045 & 1.3867 & -8.5843 \\
& Quadratic & -7.8558 & 1.5969 & -7.7746 \\
& Discrete & -7.8558 & 1.5977 & -3.0802 \\
\hline
\end{tabular}

${ }^{*}$ Results for $\mathrm{X}_{2}=6$

${ }^{\text {a }}$ Heterogeneity in $\omega$ is modelled through a MXL model

b $\omega$ is erroneously considered constant, so a MNL model is estimated 
MSE in the estimated value of a hypothetical change in $X_{1}$ from the BAU level (6) to a level of 2 under each assumption about $\omega$ when the true Cost parameter presents scale heterogeneity and the true utility specification is non-additive (over 1,000 repetitions)

\begin{tabular}{|c|c|c|c|c|}
\hline \multirow{2}{*}{$\begin{array}{l}\text { True utility } \\
\text { specification }\end{array}$} & \multirow{2}{*}{$\begin{array}{l}\text { Assumed utility } \\
\text { specification }\end{array}$} & \multirow[b]{2}{*}{ Constant $^{b}$} & \multicolumn{2}{|c|}{ Mistaken heterogeneity source } \\
\hline & & & Lognormal $^{\mathbf{a}}$ & Triangular $^{\mathrm{a}}$ \\
\hline \multirow{3}{*}{ Cobb-Douglas 1} & Linear & 225.938 & 3.218 & 29735.351 \\
\hline & Quadratic & 222.204 & 2.777 & 52709.992 \\
\hline & Discrete & 222.204 & 2.777 & 7758.046 \\
\hline \multirow{3}{*}{ Cobb-Douglas 2} & Linear & 67.379 & 2.500 & 1951.486 \\
\hline & Quadratic & 61.782 & 3.152 & 768.642 \\
\hline & Discrete & 61.782 & 3.154 & 3192.474 \\
\hline
\end{tabular}

${ }^{*}$ Results for $\mathrm{X}_{2}=6$

${ }^{\text {a }}$ Heterogeneity in $\omega$ is modelled through a MXL model

b $\omega$ is erroneously considered constant, so a MNL model is estimated

Relative bias in the estimated value of a hypothetical change in $X_{1}$ from the BAU level (6) to a level of 4 under each assumption about $\omega$ when the true Cost parameter presents scale heterogeneity and the true utility specification is nonadditive (over 1,000 repetitions)*

\begin{tabular}{|c|c|c|c|c|}
\hline \multirow{2}{*}{$\begin{array}{l}\text { True utility } \\
\text { specification }\end{array}$} & \multirow{2}{*}{$\begin{array}{l}\text { Assumed utility } \\
\text { specification }\end{array}$} & \multirow[b]{2}{*}{ Constant $^{b}$} & \multicolumn{2}{|c|}{ Mistaken heterogeneity source } \\
\hline & & & Lognormal $^{\mathrm{a}}$ & Triangular $^{\mathrm{a}}$ \\
\hline \multirow{3}{*}{ Cobb-Douglas 1} & Linear & -0.4100 & -0.0213 & -0.5670 \\
\hline & Quadratic & -0.4676 & -0.0241 & -0.6239 \\
\hline & Discrete & -0.4676 & -0.0241 & -0.9612 \\
\hline \multirow{3}{*}{ Cobb-Douglas 2} & Linear & -0.6374 & 0.4190 & -0.6793 \\
\hline & Quadratic & -1.0406 & 0.0873 & -0.6973 \\
\hline & Discrete & -1.0405 & 0.0874 & -0.2877 \\
\hline
\end{tabular}

${ }^{*}$ Results for $\mathrm{X}_{2}=6$

${ }^{a}$ Heterogeneity in $\omega$ is modelled through a MXL model

${ }^{\mathrm{b}} \omega$ is erroneously considered constant, so a MNL model is estimated 
Bias in the estimated value of a hypothetical change in $X_{1}$ from the BAU level (6) to a level of 4 under each assumption about $\omega$ when the true Cost parameter presents scale heterogeneity and the true utility specification is non-additive

(over 1,000 repetitions) ${ }^{*}$

\begin{tabular}{|c|c|c|c|c|}
\hline \multirow{2}{*}{$\begin{array}{l}\text { True utility } \\
\text { specification }\end{array}$} & \multirow{2}{*}{$\begin{array}{l}\text { Assumed utility } \\
\text { specification }\end{array}$} & \multirow[b]{2}{*}{ Constant $^{b}$} & \multicolumn{2}{|c|}{ Mistaken heterogeneity source } \\
\hline & & & Lognormal $^{\mathrm{a}}$ & Triangular $^{\mathrm{a}}$ \\
\hline \multirow{3}{*}{ Cobb-Douglas 1} & Linear & -6.6187 & 0.3433 & -9.1537 \\
\hline & Quadratic & -7.5493 & -0.3887 & -10.0735 \\
\hline & Discrete & -7.5494 & -0.3885 & -15.5182 \\
\hline \multirow{3}{*}{ Cobb-Douglas 2} & Linear & -2.8937 & 1.9019 & -3.0835 \\
\hline & Quadratic & -4.7237 & 0.3962 & -3.1656 \\
\hline & Discrete & -4.7237 & 0.3968 & -1.3059 \\
\hline
\end{tabular}

*Results for $\mathrm{X}_{2}=6$

${ }^{\text {a }}$ Heterogeneity in $\omega$ is modelled through a MXL model

b $\omega$ is erroneously considered constant, so a MNL model is estimated

MSE in the estimated value of a hypothetical change in $\mathrm{X}_{1}$ from the BAU level (6) to a level of 4 under each assumption about $\omega$ when the true Cost parameter presents scale heterogeneity and the true utility specification is non-additive (over 1,000 repetitions)

\begin{tabular}{|c|c|c|c|c|}
\hline \multirow{2}{*}{$\begin{array}{l}\text { True utility } \\
\text { specification }\end{array}$} & \multirow{2}{*}{$\begin{array}{l}\text { Assumed utility } \\
\text { specification }\end{array}$} & \multirow[b]{2}{*}{ Constant $^{b}$} & \multicolumn{2}{|c|}{ Mistaken heterogeneity source } \\
\hline & & & Lognormal $^{\mathrm{a}}$ & Triangular ${ }^{\mathrm{a}}$ \\
\hline \multirow{3}{*}{ Cobb-Douglas 1} & Linear & 43.834 & 0.618 & 7416.648 \\
\hline & Quadratic & 57.080 & 0.705 & 13643.852 \\
\hline & Discrete & 57.080 & 0.705 & 1873.944 \\
\hline \multirow{3}{*}{ Cobb-Douglas 2} & Linear & 8.390 & 3.762 & 478.958 \\
\hline & Quadratic & 22.371 & 0.525 & 109.450 \\
\hline & Discrete & 22.371 & 0.526 & 377.298 \\
\hline
\end{tabular}

${ }^{*}$ Results for $\mathrm{X}_{2}=6$

${ }^{a}$ Heterogeneity in $\omega$ is modelled through a MXL model

b $\omega$ is erroneously considered constant, so a MNL model is estimated 
Relative bias in the estimated value of a hypothetical change in $X_{1}$ from the BAU level (6) to a level of 2 under each assumption about $\omega$ when the true Cost parameter presents scale heterogeneity and the true utility specification is nonadditive (over 1,000 repetitions) ${ }^{*}$

\begin{tabular}{llccc}
\hline True utility & Assumed utility & & \multicolumn{2}{c}{ Mistaken heterogeneity source $^{\mathbf{2}}$ specification } \\
\cline { 5 - 5 } & Specification & Constant $^{\mathbf{b}}$ & Lognormal $^{\mathbf{a}}$ & Triangular $^{\mathbf{a}}$ \\
\hline \multirow{2}{*}{ Cobb-Douglas 1 } & Linear & -0.3578 & 0.1115 & -0.5287 \\
& Quadratic & -0.3536 & 0.1206 & -0.5993 \\
& Discrete & -0.3536 & 0.1206 & -0.9604 \\
\hline \multirow{3}{*}{ Cobb-Douglas 2 } & Linear & -0.5015 & 0.9511 & -0.5590 \\
& Quadratic & -0.4486 & 0.9829 & -0.4363 \\
& Discrete & -0.4486 & 0.9831 & 0.2746 \\
\hline
\end{tabular}

*Results for $\mathrm{X}_{2}=3$

${ }^{\text {a }}$ Heterogeneity in $\omega$ is modelled through a MXL model

${ }^{\mathrm{b}} \omega$ is erroneously considered constant, so a MNL model is estimated

Bias in the estimated value of a hypothetical change in $X_{1}$ from the BAU level (6) to a level of 2 under each assumption about $\omega$ when the true Cost parameter presents scale heterogeneity and the true utility specification is non-additive (over 1,000 repetitions)*

\begin{tabular}{|c|c|c|c|c|}
\hline \multirow{2}{*}{$\begin{array}{l}\text { True utility } \\
\text { specification }\end{array}$} & \multirow{2}{*}{$\begin{array}{l}\text { Assumed utility } \\
\text { specification }\end{array}$} & \multirow[b]{2}{*}{ Constant $^{b}$} & \multicolumn{2}{|c|}{ Mistaken heterogeneity source } \\
\hline & & & Lognormal $^{\mathbf{a}}$ & Triangular $^{\mathrm{a}}$ \\
\hline \multirow{3}{*}{ Cobb-Douglas 1} & Linear & -10.6161 & 3.3079 & -15.6860 \\
\hline & Quadratic & -10.4914 & 3.5766 & -17.7789 \\
\hline & Discrete & -10.4914 & 3.5766 & -28.4933 \\
\hline \multirow{3}{*}{ Cobb-Douglas 2} & Linear & -3.3111 & 6.2802 & -3.6908 \\
\hline & Quadratic & -2.9624 & 6.4903 & -2.8812 \\
\hline & Discrete & -2.9623 & 6.4912 & 1.8132 \\
\hline
\end{tabular}

${ }^{*}$ Results for $\mathrm{X}_{2}=3$

${ }^{\text {a }}$ Heterogeneity in $\omega$ is modelled through a MXL model

b $\omega$ is erroneously considered constant, so a MNL model is estimated 
MSE in the estimated value of a hypothetical change in $X_{1}$ from the BAU level (6) to a level of 2 under each assumption about $\omega$ when the true Cost parameter presents scale heterogeneity and the true utility specification is non-additive (over 1,000 repetitions)

\begin{tabular}{|c|c|c|c|c|}
\hline \multirow{2}{*}{$\begin{array}{l}\text { True utility } \\
\text { specification }\end{array}$} & \multirow{2}{*}{$\begin{array}{l}\text { Assumed utility } \\
\text { specification }\end{array}$} & \multirow[b]{2}{*}{ Constant $^{b}$} & \multicolumn{2}{|c|}{ Mistaken heterogeneity source } \\
\hline & & & Lognormal $^{\mathbf{a}}$ & Triangular $^{\mathrm{a}}$ \\
\hline \multirow{3}{*}{ Cobb-Douglas 1} & Linear & 112.806 & 12.982 & 29577.486 \\
\hline & Quadratic & 110.173 & 14.872 & 52533.661 \\
\hline & Discrete & 110.173 & 14.872 & 7487.178 \\
\hline \multirow{3}{*}{ Cobb-Douglas 2} & Linear & 11.028 & 40.017 & 1891.419 \\
\hline & Quadratic & 8.844 & 42.726 & 716.498 \\
\hline & Discrete & 8.844 & 42.737 & 3172.820 \\
\hline
\end{tabular}

${ }^{*}$ Results for $\mathrm{X}_{2}=3$

${ }^{a}$ Heterogeneity in $\omega$ is modelled through a MXL model

b $\omega$ is erroneously considered constant, so a MNL model is estimated

Relative bias in the estimated value of a hypothetical change in $X_{1}$ from the BAU level (6) to a level of 4 under each assumption about $\omega$ when the true Cost parameter presents scale heterogeneity and the true utility specification is nonadditive (over 1,000 repetitions) ${ }^{*}$

\begin{tabular}{|c|c|c|c|c|}
\hline \multirow{2}{*}{$\begin{array}{l}\text { True utility } \\
\text { specification }\end{array}$} & \multirow{2}{*}{$\begin{array}{l}\text { Assumed utility } \\
\text { specification }\end{array}$} & \multirow[b]{2}{*}{ Constant $^{b}$} & \multicolumn{2}{|c|}{ Mistaken heterogeneity source } \\
\hline & & & Lognormal $^{\mathbf{a}}$ & Triangular $^{\mathrm{a}}$ \\
\hline \multirow{3}{*}{ Cobb-Douglas 1} & Linear & -0.3222 & 0.1731 & -0.5026 \\
\hline & Quadratic & -0.3884 & 0.1210 & -0.5680 \\
\hline & Discrete & -0.3884 & 0.1211 & -0.9554 \\
\hline \multirow{3}{*}{ Cobb-Douglas 2} & Linear & -0.3687 & 1.4706 & -0.4415 \\
\hline & Quadratic & -1.0706 & 0.8930 & -0.4730 \\
\hline & Discrete & -1.0706 & 0.8933 & 0.2402 \\
\hline
\end{tabular}

*Results for $\mathrm{X}_{2}=3$

${ }^{\text {a }}$ Heterogeneity in $\omega$ is modelled through a MXL model

b $\omega$ is erroneously considered constant, so a MNL model is estimated 
Bias in the estimated value of a hypothetical change in $X_{1}$ from the BAU level (6) to a level of 4 under each assumption about $\omega$ when the true Cost parameter presents scale heterogeneity and the true utility specification is non-additive

(over 1,000 repetitions) ${ }^{*}$

\begin{tabular}{|c|c|c|c|c|}
\hline \multirow{2}{*}{$\begin{array}{l}\text { True utility } \\
\text { specification }\end{array}$} & \multirow{2}{*}{$\begin{array}{l}\text { Assumed utility } \\
\text { specification }\end{array}$} & \multirow[b]{2}{*}{ Constant $^{b}$} & \multicolumn{2}{|c|}{ Mistaken heterogeneity source } \\
\hline & & & Lognormal $^{\mathbf{a}}$ & Triangular $^{\mathrm{a}}$ \\
\hline \multirow{3}{*}{ Cobb-Douglas 1} & Linear & -4.5288 & 2.4332 & -7.0637 \\
\hline & Quadratic & -5.4594 & 1.7013 & -7.9836 \\
\hline & Discrete & -5.4594 & 1.7014 & -13.4283 \\
\hline \multirow{3}{*}{ Cobb-Douglas 2} & Linear & -0.9614 & 3.8342 & -1.1513 \\
\hline & Quadratic & -2.7914 & 2.3285 & -1.2333 \\
\hline & Discrete & -2.7914 & 2.3291 & 0.6263 \\
\hline
\end{tabular}

${ }^{*}$ Results for $\mathrm{X}_{2}=3$

${ }^{\text {a }}$ Heterogeneity in $\omega$ is modelled through a MXL model

b $\omega$ is erroneously considered constant, so a MNL model is estimated

MSE in the estimated value of a hypothetical change in $X_{1}$ from the BAU level (6) to a level of 4 under each assumption about $\omega$ when the true Cost parameter presents scale heterogeneity and the true utility specification is non-additive (over 1,000 repetitions)

\begin{tabular}{|c|c|c|c|c|}
\hline \multirow{2}{*}{$\begin{array}{l}\text { True utility } \\
\text { specification }\end{array}$} & \multirow{2}{*}{$\begin{array}{l}\text { Assumed utility } \\
\text { specification }\end{array}$} & \multirow[b]{2}{*}{ Constant $^{b}$} & \multicolumn{2}{|c|}{ Mistaken heterogeneity source } \\
\hline & & & Lognormal $^{\mathrm{a}}$ & Triangular ${ }^{\mathrm{a}}$ \\
\hline \multirow{3}{*}{ Cobb-Douglas 1} & Linear & 20.536 & 6.421 & 7382.755 \\
\hline & Quadratic & 29.892 & 3.448 & 13606.113 \\
\hline & Discrete & 29.893 & 3.449 & 1813.447 \\
\hline \multirow{3}{*}{ Cobb-Douglas 2} & Linear & 0.941 & 14.846 & 470.775 \\
\hline & Quadratic & 7.849 & 5.790 & 100.950 \\
\hline & Discrete & 7.849 & 5.793 & 373.079 \\
\hline
\end{tabular}

\footnotetext{
*Results for $\mathrm{X}_{2}=3$

${ }^{\text {a }}$ Heterogeneity in $\omega$ is modelled through a MXL model

b $\omega$ is erroneously considered constant, so a MNL model is estimated
} 